\title{
ANTROPOLOGÍA Y CAMBIO CLIMÁTICO: RECORRIDOS, TEMÁTICAS Y PROPUESTAS
}

\author{
ANTHROPOLOGY AND CLIMATE CHANGE: TRAJECTORIES, TOPICS AND \\ PROPOSITIONS
}

\author{
José Antonio Cortés Vázquez ${ }^{1}$
}

Universidade da Coruña

\section{Humberto Miguel dos Santos Martins ${ }^{2}$ Paulo Mendes ${ }^{3}$}

Universidade de Trás-os-Montes e Alto Douro

Recibido: 28 de mayo de 2020; Aprobado: 7 de julio de 2020

\begin{abstract}
Cómo citar este artículo / Citation: Cortés Vázquez, José Antonio, Humberto Miguel dos Santos Martins y Paulo Mendes. 2020. «Antropología y cambio climático: recorridos, temáticas y propuestas». Di sparidades. Re vista de An tropología 75(2): e015. doi: <https://doi.org/10.3989/dra.2020.015>.

RESUMEN: El cambio climático se ha convertido en una de las principales problemáticas a la que nos enfrentamos hoy y el eje sobre el que giran nuevas políticas, valores, intereses, horizontes y ansiedades. Aunque los estudios antropológicos del cambio climático se encuentran aún en ciernes, están generando una serie de replanteamientos y abriendo un conjunto de líneas de estudio que no solo nos permiten un conocimiento más complejo, situado y simétrico sobre este fenómeno, sino que además sitúan a la antropología en una posición privilegiada de cara a participar en estudios multidisciplinares. Urge por lo tanto realizar una revisión de estos trabajos a nivel internacional, con el objetivo de detallar los principales aportes y líneas de investigación desarrolladas, los interrogantes y respuestas planteadas y el diálogo establecido con otros campos de estudio. Comenzamos con un recorrido por los orígenes y las distintas fases y campos por las que se han extendido los trabajos antropológicos sobre el cambio climático, con especial atención a la literatura hispana y lusófona. Seguimos con sus principales reivindicaciones y planteamientos y los ejes temáticos más consolidados, así como los emergentes. Terminamos con una serie de reflexiones sobre los nuevos escenarios que trae consigo el cambio climático, sus implicaciones para los fundamentos epistemológicos de la antropología y algunas propuestas sobre posibles direcciones que tomar en el futuro.
\end{abstract}

PALABRAS CLAVE: Antropología; Cambio climático; Medio ambiente; Clima.

1 Correo electrónico: j.a.cortes@udc.es. ORCID iD: <https://orcid.org/0000-0003-3566-7924>.

2 Correo electrónico: hmartins@utad.pt. ORCID iD: <https://orcid.org/0000-0003-3095-9784>.

3 Correo electrónico: pmendes@utad.pt. ORCID iD: <https://orcid.org/0000-0002-6567-9398>. 
ABSTRACT: Climate change has become a major contemporary problem that exerts a strong influence on new policies, values, interests, horizons and anxieties. Although anthropology has only begun to pay attention to this phenomenon in recent years, it is opening up a whole new set of research fields that not only provide us with a more complex, situated and symmetric understanding of climate change, but also situate anthropology in a privileged position in order to participate in multi-disciplinary dialogues. This paper reviews the main international literature in the anthropology of climate change, with particular attention to the work available in Spanish and Portuguese, in order to trace its main topics, advances and challenges, as well as the connections with other key fields of anthropological enquiry. We begin this paper by describing the origins, past and present trajectories of this literature. We then outline the main propositions, topics and ideas that nowadays dominate the field. The paper ends with some reflections about the implications of the phenomenon of climate change for our discipline and outlines several suggestions for future developments.

KEYWORDS: Anthropology; Climate change; Environment; Climate.

Copyright: (C) 2020 CSIC. Este es un artículo de acceso abierto distribuido bajo los términos de la licencia de uso y distribución Creative Commons Reconocimiento 4.0 Internacional (CC BY 4.0).

\section{INTRODUCCIÓN}

El cambio climático se ha convertido en un reto sin precedentes para los seres humanos, amenazando con generar -e incluso generando ya- impactos sociales de magnitudes catastróficas, tanto para las generaciones presentes como las futuras. Sin embargo, la complejidad de este fenómeno (su escala global, efectos locales y distribución extremadamente desigual e injusta) dificulta enormemente su comprensión, gestión y control (Chakrabarty 2009; Hastrup y Skrydstrup 2013). Vivimos en la antesala de un nuevo mundo -o nuevos mundos- que, mas que por venir (Danowski y Viveiros de Castro 2014), están ya prácticamente aquí (IPCC 2018), lo que nos obliga a enfrentarnos a unos contextos insólitos -o tomando prestado un término de la ecología y la geociencia, unos contextos no-análogos (Alexiades 2018)-, marcados por nuevas guerras, hambrunas, pobreza, migraciones, muertes y enfermedades, y/o por el agravamiento de las ya existentes, por causa del cambio climático. Unos fenómenos que se van a suceder a escalas espaciales y temporales de gran complejidad, dificultando su predicción y modelado, lo que hace inviable cualquier estrategia de mitigación o adapta- ción a escala global del tipo de las que se han venido implantado hasta la fecha (Arora 2019). Se espera además que estos impactos perduren incluso una vez hayan desaparecido las principales causas que los generaron (sean estas derivadas del capitalismo, la industrialización o, en general, la modernidad), lo que nos sitúa también ante una trascendencia que obliga a replanteamientos profundos no solo a nivel de acción política, sino también de generación de conocimiento y experiencia individual.

La antropología social llega relativamente tarde al estudio de los impactos sociales de las alteraciones climáticas y del cambio climático ${ }^{4}$, al menos si la comparamos con otras disciplinas afines, como la sociología o la geografía humana (Pardo 2007) y muy por detrás de otras que hoy ostentan una posición hegemónica, como la economía (Fletcher 2012; Dunlap y Sullivan 2019). De hecho, este campo de estudio solo ha empezado a popularizarse y a demandar una voz propia en la última década (Baer y Singer 2018) ${ }^{5}$, aunque es cierto que ya existían algunos trabajos puntuales desde la década de 1980. En este sentido, ¿qué aporta la antropología a la cuestión del cambio climático? ¿Sobre qué bases teóricas y empíricas se sustenta un análisis antropológico de un fenómeno de una escala y magnitud tan amplias? ¿En qué sentido son (o pueden ser vistas) las alteraciones climáticas como un hecho social (total)? ¿En qué dirección replantea la antropología unos debates hasta ahora dominados por otras ciencias? ¿Cómo abordar los discursos visibilizados y producidos sobre la salud del planeta desde disciplinas como la climatología o la geofísica, sin caer en el relativismo negacionista? ¿Puede plantear alternativas a las estrategias de mitigación y adaptación hegemónicas regidas por la economía y las leyes del mercado? ¿Y cómo le afecta

4 Los términos cambio o alteraciones, si bien parecieran sinónimos - y así lo planteamos en este artículo-, guardan sin embargo algunas diferencias que conviene señalar. Para algunos especialistas en climatología, el término alteración (climática) tiende a estar asociado a escalas temporales mayores -estructurales- y el de cambio a escalas temporales (de percepción) más inmediatas o coyunturales.

5 Sin olvidar, por supuesto, los estudios en el ámbito de una antropología física-biológica en paleoclimática - a los que por razones de espacio y de objetivos de este artículo no nos vamos a referir. $\mathrm{O}$ incluso también a algunos clásicos en antropología e historia del pensamiento social (de Montesquieu a Mauss, o Vergílio Taborda u Orlando Ribeiro en Portugal en el ámbito de una geografía humana) (ver más en Dove 2014). 
a la antropología en sí misma el fenómeno del cambio climático?

El propósito principal de este artículo es establecer una cartografía de lo que podríamos llamar Antropología del Cambio Climático que nos sirva de guía para reflexionar sobre estas distintas preguntas con base en los diversos trabajos realizados hasta la fecha. Con esto lo que buscamos en último término es animar a que la antropología se involucre más de lleno en este debate, lo enriquezca y complejice, incorporando la cuestión del cambio climático en sus análisis no como una cuestión de fondo de otros fenómenos, sino problematizándolo y convirtiéndolo en su objeto de estudio a través de las muchas y diversas perspectivas teóricas que caracterizan a la disciplina. Al fin y al cabo, esta problemática no solo conecta con la disolución de las dicotomías naturaleza-sociedad que plantean las nuevas ecologías (Biersack 1999), como la ecología política, y la necesidad de abordar el análisis de distintas formas de vulnerabilidad para poder comprender el cambio climático. La cuestión climática en antropología está también relacionada con la ampliación de la escala de lo social: su alcance de estudio va más allá de lo directo y obviamente humano. Desde escalas de nanovirus hasta escalas exoplanetarias (ej. basura espacial), el terreno de la antropología se expande y abarca todo lo que no es necesariamente una influencia antropogénica o puede determinar el curso de las experiencias y las condiciones de ser humano. Así, el estudio del cambio climático nos lleva a que la vida humana no depende solo de una política social micro y macro, sino, sobre todo, de una micro y macropolítica de todo lo biótico y abiótico ${ }^{6}$.

Pese a que, como decimos, la popularización de la cuestión climática llega tarde a la antropología frente a otras disciplinas, encontramos algunas referencias reseñables que se remontan incluso a los años ochenta (Kellogg y Mead 1980) y noventa del siglo XX (Rayner y Malone 1998). Es de destacar también el ante-

6 En última instancia, la necesidad de indagar en este campo es también una demanda que nos viene de fuera. La invitación a las ciencias sociales, y a la antropología en particular, a participar activamente y apropiarse de este objeto de estudio se basa también en el reconocimiento expreso hecho por instancias de gobernanza global (por ejemplo, Naciones Unidas) de que nos enfrentamos a fenómenos complejos cuyo abordaje en ningún modo podrá ser mono-disciplinario: <http://www.worldsocialscience. org/documents/relatorio-mundial-sobre-ciencias-sociais. $\mathrm{pdf}>$. cedente que suponen algunas tradiciones de estudio como la antropología del clima y de los riesgos naturales (Soares y García 2014) o la cercanía con los debates en torno al Antropoceno (Chakrabarty 2009; Alexiades 2018; Cometti 2020). Aún así, se trata de un campo que en gran medida está aún por explorar, con una enorme potencialidad teórica y metodológica (Mendes 2013; Eriksen 2016; Parente y Curi 2017; Almeida 2019; Taddei y Haines 2019) y que puede desembocar en cruces disciplinares muy fructíferos (Barnes et al. 2013).

De forma sintética, podemos identificar hasta la fecha tres tipos de estudios en la antropología del cambio climático. Por un lado, los dirigidos a conocer la percepción, experiencia directa y los impactos de fenómenos relacionados con las alteraciones climáticas en distintas comunidades (rurales, campesinas, urbanas, indígenas) y su relación con diversas formas de explotación, exclusión social y vulnerabilidad. Por otro, el análisis del significado y sentido que otorgan estas comunidades a los nuevos fenómenos climáticos y las estrategias de adaptación que plantean. Por último, destacan también las investigaciones centradas en el surgimiento de un nuevo entramado de gubernamentalidad dirigido sobre todo a la mitigación y adaptación al cambio climático, las lógicas y sistemas de producción de conocimiento experto que lo sustentan y la contestación y alternativas que plantean distintos colectivos y movimientos sociales.

Así, podemos decir que predominan tres corrientes teóricas distintas, pero entremezcladas: la ecología cultural (¿cómo distintas culturas forman un elemento clave en la relación de los seres humanos y las alteraciones climáticas?); la fenomenología cultural (¿qué distintas visiones e interpretaciones del cambio climático existen y cómo diversifican y problematizan la forma hegemónica occidental de entender el clima?); y la ecología política (¿cómo las relaciones de poder juegan un papel clave en la generación del cambio climático, en la configuración de distintas vulnerabilidades, la distribución desigual de impactos y responsabilidades y la configuración de respuestas de mitigación y adaptación?) (Baer y Singer 2018). A estas tres se suma más recientemente el giro ontológico (¿Qué otros cambios climáticos existen, no como explicaciones diversas de una realidad objetiva y externa, sino como ontologías múltiples que coexisten en el pluriverso?).

Tal y como describiremos en detalle a lo largo de este artículo, entre las ideas claves que empiezan a 
destacar en este campo de estudio encontramos la cuestión de cómo abordar y apropiarse de un fenómeno de la magnitud del cambio climático a la escala en la que solemos analizar la realidad. Ante esta cuestión, la antropología pone en valor su tendencia a privilegiar abordajes que reconfiguran las formulaciones y tematizaciones hegemónicas que marcan la agenda pública contemporánea. Es decir, tiene la capacidad de acceder a los temas por otras vías, con otros tiempos de investigación y a otras escalas, tanto o más necesarias (Taddei 2017; Almeida 2019). Por ejemplo, partiendo de la idea de construcción social de la realidad, entiende necesario estudiar la fabricación de discursos y narrativas que permean las agendas públicas y que gozan de determinados espacios de producción de conocimiento legítimos (esto es, científicos) como lugares de poder hegemónico (ej. el tecno-poder vinculado a determinados dominios científicos y con performatividades sociopolíticas asociadas, como argumentan Miguel y Monteiro 2014) ${ }^{7}$

Bebiendo de la antropología de la ciencia y la tecnología, y siguiendo propuestas analíticas de autores como Donna Haraway (1991), Bruno Latour (1993) y Eduardo Viveiros de Castro (1998), es la propia ciencia dominante del clima la que se convierte en objeto de estudio (Bailão 2014; Taddei 2017; Taddei y Haines 2019), sus formas de representación (especialmente a través del uso de modelos computacionales - Miguel y Monteiro 2014; Parente y Curi 2017) y el juego de realismo científico (como ontología única) que tiende a subsumir otras perspectivas, lecturas y ontologías (Danowski y Viveiros de Castro 2014; Taddei 2017; Freitas 2020). Al hacerlo, la antropología reconoce otras posibilidades analíticas, proyectivas e interpretativas sobre el clima y las alteraciones climáticas (Mesquita 2012 y 2015). Es decir, la antropología abre espacios a otras narrativas, discursos, ontologías y cosmovisiones concurrentes (cfr. Mesquita 2012 y 2015), sacando a la luz los otros cambios climáticos y haciéndolos dialogar, en la medida de lo posible, con los modelos físicos y matemáticos de las ciencias del clima. Por medio de visibilizar otras culturas, otras metafísicas (ontologías) y "mito-

7 Por el contrario, también presta atención a fenómenos como el negacionismo. Precisamente a este respecto defendemos que es tarea epistemológica y política de la antropología conocer los contextos y mecanismos generadores de este tipo de discursos y representaciones de la realidad, no simplemente ignorarlos. físicas" (Danowski y Viveiros de Castro 2014), trae a otros agentes a la arena del debate científico y de producción de conocimiento sobre el clima y las alteraciones climáticas (Ulloa 2015) ${ }^{8}$ y da voz a otros saberes y formas de producción de conocimiento y experimentación sensorial (ej. Madaleno 2012; Mesquita 2012). Indaga en las otras experiencias del cambio climático, cuestionando de paso que solo se vea como un problema sociopolítico y ambiental complejo cuando afecta al Norte Global o cuando pone en tela de juicio sus formas de vida y recursos.

Barnes et al. (2013) resumen en tres las aportaciones clave que la antropología hace a este enorme, rico y diverso debate: 1 ) El estudio de los valores y relaciones sociales que subyacen a nuestro conocimiento sobre el cambio climático y su papel en las respuestas dadas a los cambios ambientales; 2) el análisis del contexto histórico en el que surgen los debates sobre el clima; y 3) la visión holística que aporta la antropología al estudio de las relaciones entre sistemas sociales y naturales y el peso que ostentan las dinámicas sociales frente al cambio climático (2013: 541). El surgimiento de lo que podríamos llamar una antropología del cambio climático deriva por lo tanto de la constatación de la importancia de la dimensión cultural de este fenómeno y la necesidad de incluir distintas experiencias y diversos saberes sobre el clima y el tiempo atmosférico, visibilizando la construcción social de la vulnerabilidad climática con el propósito de facilitar una toma de decisiones más democrática (Ulloa 2011). Hablamos por lo tanto de un nuevo campo de estudio enfocado no solo a conocer mejor cómo las sociedades experimentan, perciben, se transforman o resisten en un contexto marcado por el cambio climático, sino también a plantear acciones más horizontales, simétricas y justas de cara al futuro.

A continuación, comenzamos nuestra cartografía planteando algunos de los antecedentes más importantes de la antropología del cambio climático, desde los inicios de la disciplina hasta nuestros días. Seguimos a continuación con un recorrido por los principales trabajos, autoras, autores e instituciones que han destacado en este campo en las últimas décadas, particularmente en España, Portugal y América Latina. $Y$ terminamos con un desglose detallado de algunos de los temas que consideramos que han recibido mayor atención. Cabe mencionar que sobre la antro-

8 Véase el ejemplo del líder y chamán yanomami, Davi Kopenawa (Dias y Marras 2019). 
pología del cambio climático existen ya algunas revisiones muy detalladas (ej. Rosewarne, Goodman y Pearse 2014; Crate y Nuttall 2016; Baer y Singer 2018; Singer 2019), pero que adolecen de una excesiva atención a estudios en lengua anglosajona, dejando a un lado la literatura producida en otras geografías e idiomas. Como se verá, gran parte de la cartografía que trazamos en este artículo se nutre de literatura hispana y lusófona, con el objetivo no solo de hacerla dialogar con esas otras revisiones ya existentes, complementándolas, actualizándolas, expandiéndolas y detallándolas, sino también para visibilizar otras aproximaciones al estudio de las alteraciones climáticas, permitiéndonos reconocer otras perspectivas, geografías y escalas.

\section{ANTECEDENTES PARA UNA ANTROPOLOGÍA DEL CAMBIO CLIMÁTICO}

Podemos encontrar los antecedentes más tempranos de una antropología del cambio climático ${ }^{9}$ en las primeras investigaciones antropológicas modernas, de Marcel Mauss a Franz Boas o Bronislav Malinowski, y su intento de entender las diversas formas de organización social como manifestaciones de diferentes contextos ecológicos/ambientales. Precisamente el clima juega un papel clave en estudios clásicos de esta época, como por ejemplo la obra de Mauss (1905) Ensayo sobre las variaciones estacionales en las sociedades esquimales (Carbonell 2001). Perdura desde entonces un interés sobre cuestiones de integración ambiental y ecológica que ha llegado hasta nuestros días (Santamarina 2008; Descola 2013), a pesar de que la crítica a conceptos como el de evolución o a la dicotomía cultura-naturaleza termina generalizándose en antropología.

El análisis del clima en antropología ${ }^{10}$, como precursor y antecedente necesario de los estudios sobre

9 Debemos aclarar que, dentro del alcance de esta revisión, y debido a la limitación formal y el alcance de este artículo, nos hemos centrados en trabajos que abordan explícitamente la cuestión del cambio climático, dejando a un lado muchas otras asociaciones temáticas posibles y obvias (por ejemplo, con el Antropoceno, la sequía, cuestiones energéticas, la erosión), que también han sido estudiadas desde la antropología. Está claro que son campos que también merecen nuestra atención y deseamos que sean objeto de revisiones detalladas en el futuro.

10 No es nuestro propósito hacer una revisión exhaustiva de cómo se ha abordado históricamente la cuestión del clima cambio climático, sigue una trayectoria paralela a la del conjunto de la antropología ecológica/ambiental (para una síntesis, ver Santamarina 2008). Como sucede con muchos otros aspectos de la realidad ambiental, el clima aparece tradicionalmente en trabajos y textos clásicos sin ser problematizado ni teorizado. No deja de estar ahí (¿quién no se acuerda, por ejemplo, de los documentales de Robert Flaherty?) y de definir la ecología cultural (y hoy política) de sociedades y grupos. Es un invariable natural y universal y no una construcción social. No es hasta los años 90 del siglo XX, en el contexto de las nuevas ecologías (histórica, simbólica, política, fenomenológica), que empieza a haber una problematización del clima como construcción social, diluyendo el dualismo cartesiano que lo relegaba al dominio ontológico de lo natural (cfr. Descola y Pálsson 2001).

Así, como resumen Katz y Lammel (2008), podemos decir que han existido históricamente tres aproximaciones distintas a la relación entre cultura y clima: los análisis deterministas, que abordan la diversidad cultural como resultado de la diversidad climática; los co-evolucionistas, que asocian una influencia y relación mutua entre cultura y clima; y la ecología simbólica, de raíz estructuralista e idealista, que entiende que las poblaciones se adaptan a su entorno solo a través de la semántica y la simbología. Más adelante veremos cómo los estudios sobre cambio climático amplían estos marcos de análisis, al facilitar, por ejemplo, la incorporación de los planteamientos generados a raíz del giro ontológico (González y Carro 2016).

De forma breve, autoras como Ulloa (2011) y Rossbach (2014) defienden que la antropología del clima, propiamente dicha, se ha centrado principalmente en estudiar la relación compleja entre cultura y clima: cómo hemos entendido históricamente las condiciones atmosféricas, los ciclos climáticos y alteraciones imprevisibles, internalizándolas a menudo a través de cuentos populares, prácticas religiosas o mitos (ej. Sillitoe 1993; Goloubinoff, Katz y Lammel 1997; Acosta 2002). Como forma de conocimiento ambiental y adoptando una perspectiva del habitar

en antropología. Existen magníficos y extensos trabajos editados previamente que abordan estos precedentes (Goloubinoff, Katz y Lammel 1997; Ulloa 2011). Por el contrario, sí nos interesa hacer una pequeña síntesis de cara a establecer las bases sobre las que se desarrolla la antropología del cambio climático. 
basada en el trabajo de Ingold (2000), podemos definir que «las concepciones sobre el tiempo atmosférico y el clima se vinculan con conocimientos que no se transmiten como normas o fórmulas, dado que están atravesados por la experiencia cotidiana, habitan lugares reconocidos y están embebidos en prácticas específicas relacionadas con las condiciones climáticas en momentos particulares» (Ulloa 2011: 34). Esta autora identifica cuatro temáticas específicas que han copado gran parte del trabajo en la antropología del clima en las últimas décadas: los efectos de desastres naturales -lluvias, huracanes, inundaciones, erupciones volcánicas y terremotos, entre otros- sobre distintas poblaciones; cómo determinadas condiciones sociales y culturales se vinculan con riesgo, vulnerabilidad, resiliencia y adaptación a estos desastres; la etno-meteorología y la diversidad de saberes sobre el clima; y los puntos de encuentro y desencuentro entre conocimientos ambientales tradicionales $y$ formas de conocimiento experto.

Algunos de estos temas han tenido más relevancia que otros, al menos en la literatura antropológica hispana y lusófona. Por ejemplo, destaca el diálogo con la antropología del tiempo: cómo los ciclos que rigen la vida social a través de su calendario ritual reflejan y conectan con los ciclos climáticos (Carbonell 2001). Tal diálogo es la base, por ejemplo, del análisis de ecología cultural entre distintas comunidades indígenas mexicanas planteado en la obra editada por Katz y Lammel (2008). Estos trabajos indagan en la continuidad e indisociabilidad de la noción de clima y tiempo a través de la complementariedad y el reflejo de los ciclos climatológicos (por ejemplo, las estaciones) con los calendarios religiosos y el tiempo cíclico sobre el que se construyen muchos ritos de comunidad. Análisis similares ha planteado Acosta (2002) en el caso de Extremadura; y Villela (1997) y Hémond y Goloubinoff (1997) de nuevo para el caso de México.

Destaca también el estudio antropológico de la diversidad de significados otorgados a distintos fenómenos atmosféricos: las diferencias, por ejemplo, entre rayos y centellas o entre tipos de vientos más fríos, más cálidos, que roban almas, que secan cosechas o casas (Nates-Cruz y Serón 1997; Katz 1997). Esta línea, más de tipo fenomenológico y muy profusa en estudios, muestra una gran diversidad y continuidad en el tiempo, encontrándonos con trabajos clásicos como el de Bonfil Batalla (1964) sobre los "graniceros" de la Sierra Nevada de México, hasta otros mucho más contemporáneos centrados en distintas visiones sobre el viento en comunidades turísticas costeras en España (Corbacho 2011).

Algunos de estos estudios diferencian entre culturas climáticas de comunidades más vinculadas al medio (tradicionales) y más desapegadas (modernas) y destacan que en las tradicionales es común otorgarle al clima el carácter de impredecible e incontrolable; algo que ha sido re-interpretado en los últimos años, desde posturas funcionalistas, como una herramienta cultural de protección frente a condiciones climáticas adversas. Se sitúan en esta línea los trabajos incluidos en el doble volumen monográfico editado por Goloubinoff, Katz y Lammel (1997), donde el conocimiento del clima entre comunidades campesinas e indígenas de México viene asociado a lo trascendente y a escalas superiores de poder, siendo interpretado esto como una forma de protección frente a alteraciones climáticas y diferenciándose así de una visión occidental y moderna del clima como comportamientos estadísticos de la atmósfera y como un otro exterior que rodea lo humano (ver más en Rossbach 2019). Veremos más adelante que mismas interpretaciones se retoman para defender marcos culturales más idóneos a la hora de abordar una política global del cambio climático (Heyd 2011).

\section{CONSTRUYENDO UNA ANTROPOLOGÍA DEL CAMBIO CLIMÁTICO}

Aunque la antropología del cambio climático no se populariza hasta bien entrado el siglo XXI, el antecedente más temprano se remonta décadas atrás, a la conferencia organizada en 1975 por William W. Kellogg (físico y meteorólogo) y Margaret Mead, que dio origen al volumen The Atmosphere: Endangered and Endangering (1980). M. Mead escribía lo siguiente en la introducción de las actas de la conferencia:

\footnotetext{
Nos enfrentamos a un periodo en el que la sociedad debe tomar decisiones a una escala planetaria. Enormes cargueros surcan los mares, aviones supersónicos cruzan el cielo y explosiones nucleares resuenan por todo el mundo (tanto físicamente como en nuestras conciencias). Si en el pasado, mientras un continente entero podía verse asolado por una plaga o por terremotos el resto del mundo podía permanecer ajeno a tal desastre, las catástrofes naturales y medioambientales de hoy afectan a la humanidad entera - interconectada como está,
} 
pero incapaz de actuar políticamente de forma conjunta ${ }^{11}$.

Entre otras cuestiones, Mead abogaba por una relación más estrecha entre las ciencias naturales y sociales; por economías en las que los derechos de emisión de polución estuvieran controlados; y por establecer lazos más fuertes entre ciencia y decisiones políticas ${ }^{12}$. La conferencia fue precursora de muchas líneas de análisis y de políticas que hoy están asentadas. Sin embargo, no es hasta la entrada de la problemática del cambio climático de lleno en la agenda internacional, a partir de la década de 1990, que comienza a aparecer un número cada vez mayor de trabajos en este campo.

En este sentido, podemos trazar una genealogía con tres periodos fundamentales que coinciden con una evolución conceptual de lo que es la naturaleza (y los fenómenos naturales) y que, en consecuencia, están basadas en perspectivas distintas sobre la relación humano-nohumano y biótico-abiótico. El primer periodo se caracteriza por la no problematización del clima como construcción social y dura hasta los años 80 del siglo XX. El segundo destaca por la politización y socialización del clima tras la creación del IPCC en 1988, la publicación del informe Brundtland en 1987 y la celebración de la Conferencia de Río en 1992. Por último, el tercer periodo está marcado por la financiarización del clima (a partir de políticas neoliberales que buscan otorgar valor de mercado al medio ambiente, popularizadas desde los inicios del siglo XXI), junto con su reconocimiento como construcción cultural. En cada una de estas fases podemos anclar no solo un posicionamiento teórico específico de la antropología, sino también una preponderancia de diferentes disciplinas.

11 Traducción nuestra. A pesar de lo premonitorias que puedan parecernos hoy sus palabras, esta conferencia no tuvo gran resonancia. Acontecía en plena crisis del petróleo de 1973, y, tal vez, la problemática biografía de M. Mead o las críticas de Derek Freeman (1983) sobre su trabajo -negado por Paul Shankman (2009)-, expliquen también por qué pasara desapercibida. Se sumaban también los fuertes ataques de los sectores conservadores de la sociedad estadounidense, que no solo consideraban a la antropóloga una peligrosa amenaza para los valores conservadores (en particular aquellos relacionados con las prácticas sexuales y educacionales), sino también una izquierdista que cuestionaba los modos de producción capitalista.

12 El Panel Intergubernamental sobre Cambio Climático (IPCC) no se constituirá hasta 1988, trece años después de la conferencia de Kellogg y Mead.
En el primer periodo, el clima y los fenómenos naturales aparecen asumidos como variables naturales explicativas de los comportamientos y las opciones humanas y sociales. Es el terreno para las ciencias naturales. En el segundo período, encontramos estudios de base sociológica (muchos en diálogos interdisciplinares con la economía, la geografía y las mencionadas ciencias naturales y exactas). Se asume la construcción social del clima y se verifican las políticas, las ideologías dominantes y los marcos de intervención. El foco se pone en las geografías y perspectivas occidentales, con conceptualizaciones dominantes en términos de riesgos y vulnerabilidades de las poblaciones occidentales. El tercer periodo define no solo esa apropiación financiera del clima antes señalada (de los recursos, del calentamiento global, de los mercados de carbono, entre otros), sino que también abre espacio al reconocimiento de que el clima y las alteraciones climáticas son vividas de forma diferente por distintos grupos humanos y que no son solo los especialistas de la ciencia moderna occidental los que tienen conocimiento válido sobre estos temas. Es un periodo en el cual los/ as antropólogos/as comienzan a multiplicar sus estudios de base etnográfica orientados a la problematización de este objeto en término de su construcción cultural y valorando nuevos terrenos y percepciones/ experiencias. El clima se vuelve no solo algo que es culturalmente vivido, sino también un fenómeno transformador subjetiva e identitariamente.

Sin embargo, aunque la popularización de la cuestión del cambio climático en la agenda pública facilita que llegue a la literatura antropológica, en muchos casos lo hace solo como nuevo contexto de otros fenómenos sociales analizados. Es decir, hablamos de numerosos estudios que solo parcialmente y de soslayo empiezan a mencionar en las últimas décadas el cambio climático como marco de otros fenómenos analizados, o bien como un horizonte nuevo al que están sujetos estudios que, por otro lado, no se centran en la cuestión del cambio climático propiamente dicha, sino que intuyen su importancia y repercusión en diversas cuestiones, como la vulnerabilidad económica en poblaciones del norte de Marruecos (Haouari 2014), los desplazamientos y movilidad de poblaciones indígenas amazónicas (Alexiades y Peluso 2016), la relación con proyectos de desarrollo en el Chaco argentino (Rodríguez Mir 2011), la actividad de los molineros de la Alpujarra (Ordóñez Vergara 1993) o las disputas entre agricultores y administración pública en espacios protegidos andaluces (Cortés Vázquez 2012). 
Así, no toda la literatura antropológica que menciona el cambio climático lo aborda como problema y objeto de investigación. Un hito destacado en este sentido es la constitución en 2010 de la Global Climate Change Task Force (GCCTF) por parte de la Asociación Americana de Antropología (AAA), con el objetivo de estimular los estudios antropológicos en torno a las alteraciones climáticas, asumiendo y retomando el legado de M. Mead. El primer informe técnico de la GCCTF se publica en 2014, con el título Changing the Atmosphere: Anthropology and Climate Change (Fiske et al. 2014). En él se defiende una serie de aproximaciones y líneas de trabajo posibles para la antropología del cambio climático, tanto puramente antropológicas como transdisciplinares. Traza también una breve genealogía del interés de la antropología (anglosajona) por este tema y se insiste en la relevancia de la etnografía. En esta misma línea y de igual importancia, se publica en 2013 en la revista Nature el artículo/manifiesto encabezado por Jessica Barnes Contribution of Anthropology to the Study of Climate Change.

En este mismo periodo de tiempo se lleva a cabo en España el primer trabajo de antropología sobre cambio climático: el proyecto de investigación Percepción del cambio climático en la Comunidad Valenciana, de Beatriz Santamarina (Santamarina 2012). Poco antes ya se habían iniciado los primeros estudios en sociología (Pardo 2007), centrados en la percepción del cambio climático y calentamiento global a nivel de ciudadanía, de forma parecida a las encuestas de opinión que por entonces se plantean desde distintos gobiernos y organismos gubernamentales. A partir de ahí son la gente de sociología quienes van a tomar la batuta en los estudios sociales del cambio climático en España, sobre todo alrededor del grupo de investigación Sociología del Cambio Climático y el Desarrollo Sostenible, de la Universidad Carlos III, y de la Red Sociología, Medio Ambiente y Sociedad (SOCMAYS).

La antropología ambiental, apenas existente en España hasta principios del siglo XXI y a pesar del impulso que da la conformación de la Red de Antropología Ambiental (ANTRAMB) en 2014, no termina por interesarse hasta la fecha en la cuestión del cambio climático. De hecho, hasta el 2014 no aparecen las dos primeras comunicaciones sobre este tema en el congreso trienal de antropología (organizado por la antigua Federación de Asociaciones del Estado Español, FAAEE, hoy convertida en Asociación de Antro- pología del Estado Español, ASAEE). Posteriormente, en el de 2017, solo habrá de nuevo dos comunicaciones. Lo mismo sucede en los congresos de la Asociación de Antropología Iberoamericana (AIBR) celebrados en España (2015, 2016, 2018 y 2019). La ausencia casi total de problematización del cambio climático en la antropología en castellano publicada en España se compensa con la aparición de importantes trabajos en América Latina, por parte por ejemplo de Astrid Ulloa en Colombia y Lioba Rossbach de Olmos y Ernst Halbmayer en Cuba. También compensa la producción en otros idiomas, sobre todo en inglés, de antropólogas y antropólogos en instituciones españolas como, por ejemplo, Victoria Reyes García de la Universitat Autónoma de Barcelona y Mar Grau Satorras de la Universitat Oberta de Catalunya.

Tenemos que irnos a otros contextos europeos y otros idiomas para encontrar un mayor interés en la materia. Tanto es así que, en contraste con lo que sucede con los congresos de antropología antes mencionados, en el último encuentro de la Société Internationale d'Ethnologie et de Folklore (SIEF), celebrado en 2019 en Santiago de Compostela (a la que asisten sobre todo especialistas de antropología y etnología de distintos países de Europa), Thomas Hylland Eriksen y Paulo Mendes organizan un simposio sobre antropología y cambio climático con quince comunicaciones, teniendo que rechazar otras tantas por falta de espacio. En la misma línea, en 2016 el Royal Anthropological Institute organiza en Reino Unido la primera gran reunión en Europa sobre antropología dedicada exclusivamente a la antropología, clima y cambio climático ${ }^{13}$, con 55 simposios distintos ${ }^{14}$.

13 <https://nomadit.co.uk/conference/rai2016\#all>.

14 A nivel de proyectos europeos, destaca el iniciado en 2009 por Kirsten Hastrup (Universidad de Copenhague), financiado por la UE y titulado Waterworlds. Hastrup y su equipo centran la investigación en tres pilares: el derretimiento del hielo, el aumento del nivel promedio de las aguas del mar y la desertificación, siguiendo una metodología comparativa por diversas geografías (https://waterworlds. ku.dk). Poco más tarde, en 2011, Thomas Hylland Eriksen (Universidad de Oslo) comienza a dirigir el proyecto Sobrecalentamiento: las tres crisis de la globalización: una historia antropológica de principios del siglo XXI, también financiado por la UE y del que se obtienen varias etnografías sobre diferentes geografías, a través de un enfoque que considera que el cambio climático es concomitante con otros problemas, a saber, las consecuencias de la globalización, particularmente lo que denomina tres crisis, 
En Portugal, también en esa primera década del siglo XXI empiezan a aparecer y afianzarse los estudios relativos al cambio climático, comenzando por los de percepción pública, especialmente desde la sociología y la psicología social, destacando el trabajo de Luísa Schmidt en el Observatório de Ambiente, Território e Sociedade ${ }^{15}$. También encontramos abordajes sobre políticas ambientales, energía y recursos naturales que remiten de algún modo a la cuestión del cambio climático (ej. Araújo y Coelho 2013). En general, en muchos casos se trata de obras pioneras que no ponen el foco directamente en el tema, sino que lo tratan de forma tangencial, enmarcándolo en trabajos sobre desarrollo sostenible y ordenación del territorio (cfr. Boeno y Ferrão 2016). Del mismo modo, aparece también en el ámbito de la ecología humana, donde se privilegia el estudio de las vulnerabilidades y de los riesgos y de la capacidad adaptativa de poblaciones en distintos casos de estudio (cfr. Ventura 2008; Carvalho 2009). Aunque apoyados en enfoques cuantitativistas, son estudios y proyectos que intentan dar voz a distintos actores locales ${ }^{16}$.

También destacan en Portugal trabajos desde la ecología política que relacionan la cuestión del cambio climático con el estudio de fenómenos complejos y globales, como la globalización. Así, por ejemplo, Erikssen (2016) establece no solo la necesidad de que la antropología contribuya desde localizaciones específicas y concretas al estudio de las alteraciones climáticas, sino que pone el foco también en temas como la globalización, el neoliberalismo y los modos como los Estados (no) lidian con fenómenos que dificultan la vida y la supervivencia de distintos colectivos. Usando la metáfora del sobrecalentamiento, dirige sus ataques a una globalización desenfrenada y los procesos de financiarización, abordando cuestiones de imprevisibilidad, aleatoriedad e incertidumbre (Amorim 2015). El tema de la gobernanza del clima y las políticas estatales y transnacionales también ocupa cierto espacio (Schmidt et al. 2012) ${ }^{17}$.

ambientales, económicas y de identidad (https://www. sv.uio.no/sai/english/research/projects/overheating).

15 <http://observa.ics.ulisboa.pt/> (accedido el 7/4/2020).

16 Este tipo de enfoque es especialmente común en países latinoamericanos como Brasil (cfr. Paz 2012; Seixas et al. 2014; Litre y Bursztyn 2015; Brandão 2019; Arbarotti y Martins 2019).

17 Véase el ejemplo en Portugal de la colaboración entre la sociología y la geofísica, protagonizado por los equipos de Luísa Schmidt y Filipe Duarte Santos.
Dentro de Portugal, el colectivo de referencia se encuentra ligado al Centro em Rede de Investigação em Antropologia (CRIA), y particularmente al grupo Desafios Ambientais, Sustentabilidades e Etnogra$\mathrm{fia}^{18}$, que aglutina a un conjunto de antropólogas y antropólogos con larga trayectoria en estudios ambientales ${ }^{19}$. Uno de los temas que trabajan es precisamente el de Alterações climáticas, quotidiano $e$ processos globais, línea en la que se inscribe, por ejemplo, el trabajo de Paulo Mendes, uno de los antropólogos de ese grupo que más pronto empezó a trabajar la cuestión climática, a través de su tesis doctoral (2013) y de su trabajo de campo en los años 90 del siglo XX en una aldea de pescadores del suroeste alentejano; y sobre todo de su proyecto postdoctoral Depois da natureza ou depois da cultura? Alterações climáticas, cultura e interconexão (2011-2014). Este mismo autor coordina más recientemente, conjuntamente con Thomas Hylland Eriksen y Sussanna Hoffman, el libro Cooling Down. Local Responses to Global Climate Change, aún no publicado.

Dentro de América Latina, el caso brasileño es (actualmente) muy fértil por varias razones. Debido a su enorme territorio, su constitución multi-étnica y la existencia de una multiplicidad de mitofísicas ${ }^{20}$ (ontologías), la fuerza representativa y reguladora de la selva amazónica en el tema de alteraciones climáticas / calentamiento global, la acción del gobierno brasileño actual (con un enfoque negacionista) ${ }^{21}$ y el conjunto de políticas desarrollistas-extractivistas que se han implementado a lo largo de los años en un intento permanente de modernizar Brasil (el primer y único Estado formado en y del positivismo). Por otro lado, la antropología brasileña tiene una fuerza y un

18 <http://cria.org.pt/wp/desafios-ambientais-sustentabilidade-e-etnografia/> (accedido el 31/3/2020).

19 El Programa de Doctorado en Políticas de Cambio Climático y Desarrollo Sostenible, una iniciativa conjunta entre la Universidade de Lisboa y la Universidade Nova de Lisboa y con su primera edición ya en el año escolar 2009-2010, tiene varios estudiantes desarrollando investigación sobre cambio climático, aunque casi todos los enfoques se desvían de las líneas metodológicas etnográficas.

20 Con mitofísica nos referimos a la noción utilizada por Viveiros de Castro en oposición a la de metafísica, en un sentido (casi) literal de una física (de la naturaleza) fundada o fundamentada en concepciones mitológicas. Por lo tanto, una física mitológica, una ciencia no testada y que se presenta como discurso absoluto e incuestionable.

21 Actualmente, de hecho, la antropología es objeto de persecución por parte del gobierno brasileño. 
papel mucho más reconocido en los debates y las agendas públicas del país que la antropología en Portugal o España. Esto es debido en parte por el tema indígena, pero también por la influencia internacional de autores como Eduardo Viveiros de Castro en diversas áreas, desde la antropología hasta la filosofía, pasando por el derecho y la política. En concreto, su libro junto con Deborah Danowski ¿Hay mundos por venir? Ensayo sobre los miedos y los fines (2014), plantea una lectura filosófica-antropológica (ontológica) sobre las condiciones (sociales, económicas, políticas) del mundo actual, no solo de Brasil, ante el cambio climático. Entre el catastrofismo y una lectura que revisa el perspectivismo amerindio, nos invitan a pensar no solo en el estado del planeta, en términos ambientales, sino también en el derecho a otras formas de vivir y pensar/conceptualizar el mundo. Así, el fin del mundo debido al cambio climático se relativiza por la misma circularidad del pensamiento animista amerindio. Después de todo, como nos recuerdan las autoras del libro: "jel mundo para ellos ya ha terminado varias veces!»

\section{TEMÁTICAS PRINCIPALES EN LA ANTROPOLOGÍA DEL CAMBIO CLIMÁTICO}

De forma resumida podemos destacar dos ideasfuerza en la literatura antropológica sobre cambio climático (Gibson y Venkateswar 2015). Primera, la de indagar en cómo el Antropoceno y el cambio climático nos ofrecen una oportunidad para superar dualismos sobre los que se ha construido la disciplina antropológica (Descola 2013), poniendo el foco en cómo estamos conectamos con el mundo y otros seres de forma inextricable y allanando de paso el terreno para nuevas colaboraciones multidisciplinares, que se entienden como la clave para un conocimiento más profundo y completo del cambio climático. Y segunda, la idea de cómo el cambio climático es en realidad un fenómeno múltiple y diverso, que afecta de forma distinta a colectivos y comunidades, a la vez que se entiende de forma diversa, cuestionando así formas hegemónicas de comprenderlo basadas en la ciencia moderna y motivando un diálogo más horizontal y simétrico entre distintos tipos de saberes e, incluso, distintas ontologías.

A continuación, desglosamos lo anterior en una serie de temáticas específicas, con especial atención a cómo han sido recogidas y planteadas en la literatura antropológica hispana y lusófona.

\section{Efectos del CAMBio CLIMÁtico}

Una de las temáticas centrales en los estudios antropológicos del cambio climático es la necesidad de estudiar cómo afecta a distintas comunidades en sus contextos locales. Hasta ahora, el conocimiento principal a través del que accedíamos a los nuevos escenarios socio-ecológicos generados por el cambio climático ha consistido primordialmente en descripciones a gran escala, de carácter estadístico y macro, como los recogidos en las predicciones planteadas ante un aumento de entre 1.5 y 2 grados realizadas por el IPCC (2018). Sin embargo, a través de lo que Welch-Devine, Sourdril y Burke (2020) denominan "micro-experiencias" del cambio climático y Crate (2011) como "etnografías climáticas", observamos el surgimiento de un número significativo de estudios que indagan en la variedad de impactos locales, las características y variabilidad a escala micro y las intersecciones con relaciones de poder desiguales que, a diversas escalas, generan diversas condiciones de vulnerabilidad (cfr. Adger 2006; Pascual et al. 2014).

Estos estudios nos dan acceso a un conocimiento mucho más situado y contextualizado sobre cómo se viven los cambios, los riesgos y los problemas derivados del cambio climático, cómo se les dota de significado y qué nuevos conocimientos y prácticas generan. Ejemplos de este tipo de trabajo lo tenemos en Sapiains, Ugarte y Hasbún (2019), sobre comunidades locales en la isla de Chiloé, en Chile, y en el de Ulloa (2018) entre grupos indígenas de Colombia. Más allá del interés por entender de una forma múltiple y diversa los efectos del cambio climático, que veremos en más detalle en los siguientes apartados, el estudio de estas distintas experiencias tiene una clara dimensión aplicada. Y es que la adaptación se ha convertido prácticamente en el nuevo concepto de moda, a partir del cual se está incorporando la cuestión del cambio climático a la agenda de las estrategias de desarrollo que han articulado históricamente la relación entre el Norte Global rico y el Sur Global "subdesarrollado" (Escobar 2007), sustituyendo conceptos otrora en boga, como el de desarrollo, derechos o participación (Pokrant 2016). Surge por lo tanto la necesidad de problematizar la cuestión de la adaptación y abordarla desde una visión crítica; y es aquí donde la antropología usa de nuevo sus herramientas para problematizar consensos y diversificar conocimientos.

Por ejemplo, hay trabajos que defienden la necesidad de diferenciar la cuestión de la adaptación en- 
tre ámbitos rurales y urbanos, puesto que en ellos hay relaciones de poder distintas (Sapiains, Ugarte y Hasbún 2019). También los hay que sugieren la necesidad de plantear la toma de decisión y gestión desde el nivel local, no desde lo supralocal (como suele ser la dinámica de las estrategias de desarrollo Norte-Sur), de modo que se facilite que la población se organice en torno a sus propios recursos y se implique en este tipo de políticas públicas (Soares y Vargas 2012). Otra línea abierta consiste en aplicar una perspectiva histórica que se centre en cómo las respuestas a alteraciones climáticas en el pasado entendiendo que el clima no es uniforme, estable y predecible- nos puede dar herramientas para pensar en cómo responder a situaciones futuras. Por ejemplo, centrándose en el estudio de archivos en Terrassa, Cataluña, Grau et al. (2016) describen estrategias campesinas ante situaciones climáticas adversas en el pasado que pueden ser de utilidad ante la actual emergencia climática.

Por último, poniendo el foco en el extremo opuesto, el macro, algunos estudios han empezado a indagar en los impactos que traen aparejadas las estrategias estatales y de multinacionales de adaptación al cambio climático. Con iniciativas como la promoción de fuentes de energía renovables, los medios de transporte limpios y la desmaterialización de la economía, altamente dependientes de nuevas tecnologías, se da lugar a lo que algunos han dado en llamar extractivismo verde; un fenómeno que, pese a sus objetivos supuestamente más sostenibles, reproducen las mismas dinámicas e ignoran la vinculación de determinados colectivos con el medio (TocancipáFalla, Rosero y Restrepo 2011; Franquesa 2018).

\section{CONTESTACIÓN DE POLÍTICAS DEL CAMBIO CLIMÁTICO}

Como apuntamos en la introducción, existe cierto consenso en la literatura crítica en ciencias sociales, no solo la antropológica, sobre la idea de que la gran mayoría de medidas adoptadas por gobiernos nacionales e instituciones internacionales (como la United Nations Framework Convention in Climate Change UNFCCC y el ya mencionado IPCC) para abordar los impactos del cambio climático ignoran su carácter complejo y, en concreto, su distribución desigual e injusta. Tomemos por ejemplo el tipo de estrategias e instrumentos de mercado que se han generalizado durante las últimas tres décadas, tales como Reducing Emissions from Deforestation and forest Degradation
(REDD), Payments for Ecosystem Services (PES) o Carbon Offsetting (Fletcher 2012; Dunlap y Sullivan 2019).

Tales instrumentos, sobre los que existe una extensa literatura en ciencias sociales, parecen ignorar las siguientes cuestiones: 1) Valorar la naturaleza meramente en términos económicos impide integrar cuestiones relacionadas con cómo se distribuyen los impactos y cómo influyen las relaciones de poder (Muradian et al. 2010); 2) La vulnerabilidad a los nuevos escenarios climáticos es desigual y es el resultado de distintos tipo de discriminación por cuestión de clase, raza, sexo, género o nacionalidad (Adger 2006; Pascual et al. 2014); 3) Cualquier tipo de predicción o propuesta de gestión general y universal, como pueden ser las planteadas a través de herramientas de mercado, es irreal e irrealizable (Arora 2019); 4) Nuevas estrategias de mitigación y adaptación al cambio climático requieren de la co-producción del conocimiento y de la toma decisiones sobre los riesgos y su distribución, es decir estrategias bottom-up (Jasanoff 2004; Forsyth 2014), en lugar de top-down (Felli 2019). En resumen, se argumenta que las principales estrategias puestas en marcha para abordar la cuestión del cambio climático a nivel global por instituciones tanto nacionales como internacionales, dejan de lado la relación dialéctica entre la distribución desigual de la responsabilidad y de la vulnerabilidad frente al cambio climático; ignoran las voces y perspectivas de las víctima del cambio climático; y consideran como únicamente viable aquellas soluciones basadas en la tecnología y en el mercado, como si los problemas de la actual crisis climática pudieran solucionarse con las mismas lógicas políticas y fundamentos teóricos que las causaron (Tokar 2019).

Ejemplos de estudios antropológicos de este tipo, con fuerte carga de ecología política, y donde se pone en relación el cambio climático con procesos como las políticas de desarrollo, la globalización y el neoliberalismo, los encontramos en el trabajo de Urte Frömming (2009), quien explora el tema del calentamiento global (y la fusión de los glaciares del Kilimanjaro) dando cuenta de cómo se reproducen los procesos de apropiación colonial de la naturaleza en África por parte de los turistas europeos. Otro ejemplo es el de Madalena Santos (2017), con un estudio de maestría sobre exploración de petróleo en la costa del Algarve (sur de Portugal), en el que explora las contradicciones del Estado portugués en términos de 
política energética/explotación de recursos naturales en relación con convenios internacionales sobre cambio climático y con un enfoque puesto en términos de respuestas y contestación por parte de la población local.

Podría argumentarse que un síntoma claro de por qué ha imperado el tipo de aproximaciones y visiones antes señalada se encuentra en la idea generalizada de que el cambio climático es un problema causado por todos (los seres humanos) y sufrido por todos. Este tipo de ideas subyace a la aproximación más ortodoxa a la idea de Antropoceno (Alexiades 2018); una aproximación que defiende que todos hemos contribuido por igual a generar el problema del cambio climático, que el problema es el ser humano en sí (sus características innatas, su número exponencialmente creciente, etcétera) y no un sistema de organización social determinado, como el capitalismo (Keucheyan 2014). Desde una perspectiva crítica, este tipo de idea ofrece una visión incompleta de la situación de crisis actual (Haraway 2015; Emmett y Lekan 2016; Bauer y Ellis 2018). Algunos incluso argumentan que tales narrativas son una estrategia para despolitizar el debate sobre el cambio climático, desviando la atención sobre las causas reales que lo han generado (Swyngedouw 2010). Así, según Foster, Clark y York (2009) o Klein (2014), abordar el problema del cambio climático pasa por identificar claramente sus raíces en las lógicas, la distribución de poder en el capitalismo y la justificación ideológica que otorga el proyecto de la modernidad, por el cual la esencia de los humanos es la de ser los dominadores de la naturaleza (para un análisis más elaborado del carácter histórico del cambio climático, ver Chakrabarty 2009; Emmett y Lekan 2016).

Precisamente en estrecha vinculación con este tema, nos encontramos otra línea de investigación de gran potencia: la relacionada con los procesos de contestación, acción colectiva y movilización ciudadana. A pesar de la incertidumbre que rodea a los impactos sociales del cambio climático, una cuestión parece cierta: aunque terminará afectándonos a todos de una manera u otra, indistintamente de nuestra clase, género o nacionalidad, su distribución será desigual, afectando más a quienes por lo general han contribuido menos a generarlos, como por ejemplo grupos indígenas y comunidades vulnerables en el Sur Global. De hecho, son estos colectivos los que ya sufren de forma desproporcionada los efectos del cambio climático (Singer 2019). Precisamente esta distribución desigual de los impactos se ha convertido en el argumento central de numerosos movimientos sociales que han ido surgiendo en los últimos años, generalmente conocidos como movimientos de justicia climática (Schlosberg y Collins 2014; Jafry 2019). Reivindican una distribución más equilibrada de las causas y efectos sociales del cambio climático, así como el reconocimiento de los múltiples impactos que este genera, cómo se retroalimenta con situaciones de vulnerabilidad (por clase, por género, por nacionalidad) y la necesidad de tener voz en decisiones relacionadas con la adaptación y remediación de estos impactos.

Si la visión más común y compartida de las iniciativas de mitigación y adaptación puesta en marcha por gobiernos y empresas va contra las demandas y las reivindicaciones de los movimientos de justicia climática, en particular, y de las víctimas del cambio climático, en general, no es difícil imaginar por qué han sido el objeto de críticas y rechazos durante las últimas dos décadas (Godden y Tehan 2016; Bond y Dorsey 2010). Como analizan diversas autoras y autores, estos movimientos surgieron como una reacción radical al tipo de movimiento ecologista mainstream que acabó dominando la esfera política en las últimas décadas, de la mano del proyecto neoliberal (Pettit 2004; Schlosberg y Collins 2014); críticas que se centraban en su escasa interseccionalidad y en su pragmatismo político, lo que las hacía más proclive a aceptar las reglas del juego capitalista, que a plantear un cambio radical.

Los movimientos de justicia climática, bebiendo tanto de la teoría como de la práctica de movimientos de justicia ambiental (Temper 2019) -y de sus raíces en los movimientos por los derechos civiles-, pasaron a centrar sus demandas en los principios de reconocimiento, distribución y participación (cfr. Fraser 2000) como las claves para asegurar una distribución más igualitaria y equitativa de los problemas generados por la actual crisis ecológica. Hoy se centran sobre todo en denunciar cómo los impactos climáticos y las respuestas que damos a estos poseen determinado potencial tanto para profundizar como para aliviar las desigualdades sociales (Jafry 2019).

Los estudios realizados sobre estos movimientos destacan su énfasis en impactos y experiencias locales, vulnerabilidades desiguales y la importancia de dar voz y poder a las comunidades locales (Schlosberg y Collins 2014; De la Villa 2019). Desde los efectos de inundaciones en la precariedad económica y la 
salud mental de comunidades urbanas y rurales, al drama de comunidades indígenas debido a cambios en las temporadas de nevada y la reducción del permafrost en el Ártico, pasando por migraciones y desplazamientos causados por sequías o por el agotamiento de agua dulce, el estudio de las denominadas víctimas climáticas no ha hecho más que crecer en número, a medida que estas se organizan en nuevos movimientos que demandan justicia, hasta alcanzar hoy escala global, con presencia casi constante en grandes eventos y manifestaciones como las que rodean a las periódicas Conferencia de las Partes (COP, en sus siglas en inglés), organizada por la Convención Marco de la ONU sobre el Cambio Climático (Diz 2017).

\section{ANÁLISIS DE LA GÉNESIS Y PERCEPCIÓN DEL CAMBIO CLIMÁTICO}

Otro conjunto importante de estudios en antropología del cambio climático se interroga acerca de distintos aspectos relacionados con la génesis de este fenómeno, dialogando con el debate abierto alrededor de la noción de Antropoceno, donde una de las cuestiones centrales ha sido precisamente fijar el supuesto inicio de esta nueva era geológica (Alexiades 2018; Cometti 2020). Algunos trabajos en esta línea plantean una lectura más completa, crítica y profunda que la realizada desde las ciencias naturales (cfr. Crutzen y Steffen 2003) en relación con cómo los seres humanos han interaccionado con el clima, global y local, abordando el contexto presente a través de un prisma histórico donde las alteraciones climáticas son vistas como recurrentes en el tiempo y criticando así una visión del contexto ambiental como estable y en equilibrio (y hoy, sin embargo, cambiante) heredada de la ecología clásica de Haeckel e incorporada a numerosos estudios de ecología cultural, desde Steward (1938) a Rappaport (1979). Así, trabajos como el de Grau et al. (2016), ya mencionado anteriormente, indagan en cuáles han sido las prácticas y estrategias de adaptación en contextos de alteraciones climáticas en el pasado y como estas pueden iluminar nuevas estrategias de cara al futuro, poniendo sobre la mesa la necesidad de entender el clima en su carácter siempre dinámico.

Ahondando también en el estudio del pasado en busca de una génesis del cambio climático, pero yendo mucho más allá de los siglos XIX y XX donde una lectura ortodoxa del Antropoceno suele situarlo
(Alexiades 2018), hay una segunda corriente que, combinando antropología y arqueología, se centra en determinar si los cambios a nivel de paisaje, vegetación y usos hace miles de años podrían corresponderse o no al cambio climático o a otros procesos, como la minería a gran escala y el pastoreo (Allué et al. 2007; Vicent et al. 2010). No es casual que estas indagaciones surjan precisamente del diálogo con la arqueología, pues esta ha prestado mucha más atención a la cuestión del clima como desencadenante de fines de civilizaciones (Diamond 2006). Existe gran controversia en este tipo de planteamientos, al haber autoras y autores que critican cierto determinismo al importar explicaciones propias de las cosmologías modernas y sus imaginarios sobre el fin del mundo para explicar dinámicas sociales pasadas (Marconetto y Bussi 2018; Villalobos 2018).

Encontramos una tercera corriente de estudios que examina la génesis del cambio climático desde el punto de vista subjetivo; es decir, no como un análisis de ecología cultural, sino desde la óptica del momento y modo en el que empezamos a percibir la existencia del cambio climático como una nueva realidad y un nuevo horizonte de acción. No hablaríamos tanto del análisis estadístico de la percepción del cambio climático como preocupación y los nuevos valores (ambientales) que lleva aparejado ${ }^{22}$. Se trata más bien de abordar la percepción del cambio climático desde su comprensión diversa (de efecto, de responsabilidad), dentro de las complejidades que lleva aparejado el fenómeno a través de sus múltiples escalas (Santamarina 2012). Destaca en este sentido, el trabajo coordinado por Luísa Schmidt (OBSERVA-ICS), que combina aproximaciones cuantitativas a las actitudes, comportamientos y representaciones sociales sobre el cambio climático entre los portugueses (Schmidt et al. 2018; Ferrão y Delicado 2019; Guerra et al. 2019), con análisis cualitativo de prácticas y representaciones (ej. Gomes 2019 sobre poblaciones rurales en el norte de Mozambique) e incluso con estudios aplicados al ámbito de la educación para el clima (Delicado et al. 2018).

Un argumento común en este tipo de estudios de percepción y de surgimiento de la preocupación por el cambio climático es que las comunidades locales y campesinas están más predispuestas a entender

22 Un campo con gran recorrido en sociología y cuya descripción pormenorizada se sale de los objetivos de esta cartografía. 
mejor las causas y consecuencias locales de cambios en el clima. Esto supone una oportunidad única para abordar la diversidad de experiencias situadas y locales del cambio climático, complementando y matizando así el carácter hegemónico del conocimiento experto a la hora de presentarse como vía principal y única legitimada para percibir un fenómeno de tal complejidad y amplitud (Burman 2017). En este sentido, diversas autoras y autores han hecho un esfuerzo notable por describir distintas formas de percepción del cambio climático entre comunidades locales e indígenas (Fernández Llamazares et al. 2017; Reyes García et al. 2019), prestando especial atención a comunidades ribereñas y pesqueras, que se han convertido en sujetos de estudio privilegiados (Delicado et al. 2012; Madaleno 2012; Mendes 2013; Seixas et al. 2014; Amorim 2015; Ribeiro 2015; Freitas 2020). Al vivir y habitar zonas costeras, más susceptibles y vulnerables - con mayor riesgo en términos de erosión derivado de la subida del nivel del mar- se entiende que desarrollan percepciones y una experiencia de transformación del paisaje, del territorio y del ambiente más directa y cercana.

En este sentido, Santamarina (2012) plantea algunas de las cuestiones clave para adentrarse en esta línea de trabajo: ¿Cómo se percibe el cambio climático? ¿Qué mediadores actúan en su construcción? ¿Cuáles son las fuentes de legitimidad de la percepción del cambio climático? ¿Cómo se identifican las causas y consecuencias del cambio climático en el tiempo y en el espacio? Aquí entrarían a jugar distintos factores, no solo mentales sino también corporales, nutriéndose de un corpus muy elaborado de trabajos centrados en la relación entre cuerpo, salud y ambiente, a través de nociones como la de "cuerpos permeables" (Begueria y Zafra-Aparici 2019), relacionales y abiertos al mundo que les rodea.

\section{Diversas VISIONES DEL CAMBIO CLIMÁTICO}

Otra temática que ha acaparado un número significativo de páginas de literatura antropológica es la que defiende la existencia de distintos sentidos y significados asociados al cambio climático. En esta línea debemos diferenciar dos aproximaciones distintas: la multiculturalista (distintas interpretaciones de una realidad única), más clásica y en la que nos centraremos en este apartado; y la ontológica o plurinatural, más incipiente y que desarrollaremos en próximos apartados (cfr. Descola 2013). Bebiendo de estudios sobre la re- lación entre clima y cultura (Ulloa 2011) y la etno-climatología (Mesquita 2012 y 2015), en la primera corriente se inserta por ejemplo el trabajo de Rossbach y Halbmayer (2014), que coordinan un monográfico sobre cambio climático donde indagan en las diversas formas de entender y dar sentido a eventos climáticos, especialmente aquellos relacionados con fenómenos extremos como los huracanes. Centrándose en el contexto cubano, destacan especialmente el análisis de los métodos de conocimiento del clima por parte de campesinos y sanadores (Rosario 2014) y las mejores adaptaciones que ofrece el conocimiento climático tradicional del campesinado frente a los modelos que sustentan proyectos de adaptación a nuevas especies de cultivo (González y Rubio 2014). Del mismo modo, Galván Tudela y Guevara Labaut (2014) se preguntan, ante el análisis de fenómenos extremos, si comparado con los sistemas de medición y predicción remota sobre los que se fundamenta la ecogubernamentalidad global del clima (Ulloa 2010), los patrones culturales de respuesta local son capaces de reconocer con más precisión las características climáticas locales y sus efectos, así como dar lugar a estrategias más eficientes de adaptación.

La perspectiva multicultural en la antropología del cambio climático nos lleva también a reflexionar sobre la cuestión de la temporalidad, en un doble sentido. Por un lado, en el de tiempo meteorológico, que se contrapone a la idea de clima precisamente en su escala cronológica. La etnografía, la arqueología y la microbiografía combinadas son claves para entender, por ejemplo, cómo se experimentan los cambios meteorológicos de forma diacrónica y darnos información sobre el cambio climático. Por otro lado, también nos lleva a la cuestión del tipo de temporalidad cronológica moderna -de tipo lineal, basado en la superación del pasado que ya no existe-, que trae consigo determinados mitos de final apocalíptico. Como apuntan Danowski y Viveiros de Castro (2014), deberíamos pensar también en otras cronologías, en finales que no cierran y pasados que no acaban, sino que perduran, para entender también el cambio climático. Por ejemplo, trabajos como los de Vázquez y Tovar (2018) analizan cómo, más allá de las temporalidades apocalípticas occidentales, las narrativas sobre el cambio climático penetran y se replantean dentro de otros marcos de lecturas apocalípticas propias, como por ejemplo los de las iglesias evangélicas en México.

En relación con la diferenciación de saberes, destacamos el trabajo de Mesquita (2012 y 2015), quien 
busca acceder al conocimiento tradicional de los Ashaninka y los Kaxinawá en la región del Alto Juruá en el estado de Acre (Brasil). En su tesis doctoral (2012), la autora explora visiones del mundo y percepciones entre los miembros de esos dos grupos étnicos; un enfoque y un objeto que vemos reflejado en otros trabajos etnográficos (por ejemplo, Lobo 2016). Su propuesta también nos lleva a la relación entre ciencia y conocimiento ecológico tradicional o indígena. De hecho, tanto Mesquita como también Taddei (2014, 2016 y 2017), han indagado en las contribuciones de ese conocimiento al estudio del clima y el cambio climático, planteando la antropología como mediadora entre actores y contextos que a menudo son poco comunicativos (Faulhaber 2004).

Sobre este diálogo de saberes, Rossbach (2019) muestra algunas de las barreras filosóficas que los separan y que dificultan las posibilidades de traducción y diálogo necesarios a la hora de incorporar estos otros conocimientos tradicionales o indígenas (cfr. Agrawal 1995) a los estudios del cambio climático. González Martínez et al. (2015) entretejen esta diversidad de formas de entender el cambio climático con relaciones de poder desiguales. En su estudio entre los campesinos purepechás de México critican al tecnocentrismo que domina la respuesta y aproximación global al cambio climático, como producto de relaciones coloniales que configuran la comunidad local y de situaciones de desigualdad interna dentro de comunidades de campesinos. Enfoques similares, donde se vinculan la vulnerabilidad, la marginación de conocimientos indígenas y la justicia climática, aparecen en otros trabajos, como por ejemplo Soares y García (2014) en México, Correa (2012) en el Caribe colombiano y Cometti (2020) sobre los q'ero de los Andes.

En este sentido es importante reconocer el conocimiento climático como un conocimiento corporeizado y que no existe de forma independiente de la práctica (Ingold 2000). Así, la práctica cotidiana desarrollada en contextos concretos de relaciones de poder, determinados por ejemplo por el género (Ravera et al. 2019) o por la capacidad física (Kosanic et al. 2019), va a jugar un papel clave para acceder a esta idea de conocimiento local. En otras palabras, el conocimiento local sobre el clima no se refiere a una escala concreta y por lo tanto determinada por la condición espacial (lo local), sino a la situación particular que un colectivo ocupa en el tejido social. Así, cómo detallan Ravera et al. (2019) y Ravera e Iniesta
(2017), podemos hablar de la existencia de una visión particular del cambio climático desde la perspectiva de género, sin homogeneizar a las mujeres y abordando la cuestión de género en intersección con otros procesos de constitución de las relaciones de poder con base en ejes identitarios como la clase, la etnicidad, la sexualidad o la nacionalidad (más en AroraJonsons 2011; Ulloa 2014 y 2016; Djoudi et al. 2016).

Frente a estos análisis de conocimientos climáticos indígenas y locales, vemos cómo ha ido floreciendo también el estudio de la producción del conocimiento experto, que en el nivel global compone la visión hegemónica (occidental, moderna) que tenemos del cambio climático (Burman 2017; Arora 2019). Bebiendo de los estudios de ciencia y tecnología (STS, en inglés), autoras como Ulloa (2010) en Colombia y Carabajal (2016) en Argentina, indagan en cuáles son los aspectos socio-culturales e institucionales involucrados en la producción de la información climática oficial, así como las complejidades y desafíos que surgen en el proceso de provisión de servicios climáticos.

Este tipo de indagación persigue por lo general un doble propósito. Por un lado, reflexionar sobre la construcción del conocimiento experto legítimo en el tema del cambio climático. Como apuntamos anteriormente, el cambio climático ha sido históricamente el terreno de juego de las ciencias puras (Urry 2011). Así, la antropología ha prestado atención y reflexionado sobre el modo como los/as científicos/ as ambientales y del clima se apropian política y simbólicamente de los discursos y las representaciones (Bailão 2014; Miguel y Monteiro 2014; Taddei 2017; Taddei y Haines 2019) y como los otros, los y las antropólogos y antropólogas, necesitan justificarse permanentemente para abordar estos estudios y dialogar con esas disciplinas. Estamos ante un campo en el cual se ponen en juego poderes y donde surgen cuestionamientos al nivel de la producción y del derecho a producir conocimiento (Bailão 2014; Taddei y Haines 2019). En este sentido, son espacialmente útiles las etnografías sobre cómo los dominios científicos se apropian del estudio del clima y las alteraciones climáticas, de las culturas epistémicas que los configuran y de los efectos performativos en el campo de la política, la sociedad y la ciencia (cfr. Miguel y Monteiro 2014; Taddei 2017; Fleury y Taddei 2019).

Por otro lado, surgen al hilo del debate sobre las múltiples visiones del cambio climático una serie de trabajos que se centran precisamente en la necesidad 
y las posibilidades de diálogo entre estos tipos de conocimiento y las ventajas de incorporar conocimientos climáticos tradicionales y locales, que aportan un alto nivel de detalle sobre las características del cambio climático a escala local (Fernández Llamazares et al. 2017; Reyes García et al. 2019). Como argumentan García del Amo, Mortyn y Reyes-García (2020), es cada vez más evidente la necesidad de implicar los conocimientos locales e indígenas a la hora de producir una descripción detallada a escala local de los impactos que genera el cambio climático, frente a modelos predictivos y los análisis realizados desde las ciencias físicas y naturales, que carecen de una resolución lo suficientemente detallada como para comprender cómo se desenvuelve de forma múltiple y diversa el cambio climático a escala local. Esta urgencia viene también motivada por la necesidad de pensar estrategias situadas y contextualizadas de respuesta y adaptación al cambio climático, ya que las comunidades locales han desarrollado un conocimiento sobre cómo adaptarse a condiciones climáticas cambiantes y eventos meteorológicos extremos a lo largo de la historia en su contexto local.

Indagando en la posibilidad y mecanismos para este tipo de colaboraciones, Joana Roque de Pinho (2013; Roque de Pinho y Galvin 2015) ha desarrollado un enfoque colaborativo con los masái en Kenia y los agricultores en Guinea-Bissau, trabajando a la vez con biólogos y antropólogos. Una de sus principales opciones metodológicas ha sido ofrecer cámaras a las poblaciones estudiadas para que retraten sus contextos cambiantes. La autora ha desarrollado así un trabajo en la intersección entre la antropología ambiental y la ecología política, generando formas de percepción y conocimiento local sobre el cambio climático. Su propuesta no solo es muy interesante en términos metodológicos (por el uso de medios visuales y, en particular, la foto-obtención) y epistemológica, en el contexto de varios cruces disciplinarios (antropología, ciencias ambientales, biología) y conocimiento (incluidos los locales o tradicionales), sino también en términos éticos y políticos, dentro de lo que podríamos llamar una antropología compartida o estudios colaborativos.

\section{“Otros” CAMBIOS CLIMÁticos}

Finalmente, frente a la perspectiva multiculturalista antes descrita, Danowski y Viveiros de Castro (2014) plantean la cuestión de la colonización no solo epistémica ${ }^{23}$ (Santos y Meneses 2010), sino también ontológica (Escobar 2016) en el marco del cambio climático. Critican cómo gran cantidad de estudios en la antropología del cambio climático parten de una concepción relativista que da por hecho la existencia del cambio climático como realidad objetiva, sobre la que se vierten múltiples interpretaciones culturales. Bien desde la óptica del perspectivismo de Viveiros de Castro (1998), bien desde las ontologías relacionales de Escobar (2016), un grupo destacado de autores y autoras plantea la necesidad de explorar, de una forma simétrica (Latour 1993), otros cambios climáticos no como explicaciones diversas de una realidad objetiva, sino como ontologías múltiples que coexisten en el pluriverso (Burman 2017).

Desde esta perspectiva, las lecturas hegemónicas del cambio climático adquieren el carácter de ocupación ontológica del territorio global; y las luchas y movimientos por el clima se convierten en luchas ontológicas. Parafraseando a Escobar (2016), el Mundo mundial, dentro del que se plantea el cambio climático como realidad única y universal, priva a los otros mundos (los otros cambios climáticos) de su posibilidad de existir. El cambio climático representa en sí una de las paradojas del proyecto moderno, sustentado en la separación naturaleza-sociedad, al extinguir la propia naturaleza como referente propio. Precisamente aquí radican una serie de angustias modernas, que, sin embargo, no se corresponde con cómo se entiende el cambio climático desde otras poblaciones. Danowski y Viveiros de Castro nos invitan a explorar esta cuestión desde la perspectiva de esos fines del mundo "otros": "otras" narraciones del fin del mundo, como punto constituyente de muchas culturas, y "otras" experiencias, presentes y pasadas del fin.

Por ejemplo, autores como Marconetto y Bussi (2018) indagan en el concepto nativo de seca en Catamarca (Argentina), comprendida como un estado de constante reducción de las intensidades de diversas esferas de la vida local; frente a la idea de sequía, definida en términos de déficit de agua. La seca se convierte así en un concepto que aglutina un cambio paulatino, una disminución de intensidad en distintas

23 ¿Hace cuántos años que los/las antropólogos/as vienen "denunciado" y criticado la homogeneización cultural e ideológica subyacente a los proyectos de desarrollo capitalistas promovidos desde ciertos centros geográficos, económicos y políticos? 
esferas, de la mano de cambios en estilos de vida, en tipos de trabajo, en presencia de especies silvestres, bajada en la producción agrícola, modernización de la vida y reducción de lluvias. La seca es «más-queambiental y más-que-social, traspasando lo atmosférico y lo histórico hasta desdibujar dichos tabiques» (Marconetto y Bussi 2018: 324).

Lo que plantea un abordaje simétrico de los otros cambios climáticos es romper con las tendencias naturalistas y culturalistas que han dominado la historia de la antropología (Descola 2013). En esta línea destaca, por ejemplo, la obra de Kopenawa y Albert (2013) donde hacen una lectura de cómo se experimenta el cambio climático entre los yanomami; o el trabajo de Burman (2017) en Bolivia sobre cómo se entiende el clima (por ejemplo, la ocurrencia de lluvias) a partir de relaciones políticas y diplomáticas entre seres humanos y no-humanos.

\section{SEGUIR PENSANDO EN CAMBIO(S) CLIMÁTICO(S)}

El uso del tiempo plural que sugerimos al hablar de cambio(s) climático(s) no es meramente una cuestión de estilo. Al contrario, evoca y establece mucho de lo que nos parece que son las distintas direcciones que debe tomar la antropología en este campo. Elegimos el plural para concluir este artículo porque, como hemos visto a lo largo de sus páginas, diversas son las alteraciones climáticas en curso, múltiples sus causas y consecuencias, plurales los contextos y las percepciones de sus efectos y diversas las responsabilidades y las agencialidades en este Antropoceno vivido de forma desigual. Conocimientos, apropiaciones, usos, narrativas, discursos, adaptaciones, mitigaciones, soluciones, programas... No hay soluciones únicas a este problema porque no todos sienten y presencian las alteraciones climáticas de la misma forma y, aunque en la narrativa dominante se hable de un único planeta, la verdad es que, como demuestra la antropología, este no es igual para todos.

La antropología, inmersa en su tarea histórica de convertir en plural lo que se presenta como singular, de mostrar diverso lo que tantos insisten en plantear como único y hegemónico, tiene en este terreno de estudio y en este objeto de análisis dos desafíos grandes de cara al futuro. Uno de estos desafíos está relacionado con nuevos equilibrios existenciales entre seres humanos y todo lo demás; con nuevas condiciones de existencia que obligatoriamente nos llevan a tener que pensar a otras escalas en términos de una política amplia de las cosas, reconociendo su estatus político. El segundo gran desafío es implicar la antropología en otras posibilidades etnográficas y en otras geografías de conocimiento y reconocimiento, desde lo más micro a lo más macro. Comenzando, desde luego, por el cuestionamiento fundamental sobre quien y desde donde produce conocimiento y habla sobre el cambio climático. Se preguntaba Philippe Descola años atrás: ¿Quién habla por la Naturaleza? ¿Quién es dueño de la Naturaleza? ${ }^{24}$. Del mismo modo, podemos preguntarnos hoy: ¿Quién es dueño del clima y habla por las alteraciones climáticas? Aún más: ¿En que medida las narrativas y discursos sobre las alteraciones climáticas no son ellas mismas productos de una tecnociencia que intenta dominarlo todo - cual Deus ex machina-, aun siendo ella subsidiaria de modos de vida que han acelerado el proceso natural de las alteraciones y cambios climáticos? O sea, ¿en qué medida la propia producción discursiva dominante no es en sí un producto del capitalismo dominante?

La antropología también nos muestra que estamos ante un falso dilema: una producción discursiva catastrofista por un lado y una negacionista por otro. Dos narrativas polarizadoras en una lucha por los recursos en el campo de la representación y la institucionalización del problema, que no deja espacios para otros tiempos, otras vidas y otras interpretaciones ${ }^{25}$. No se reivindica aquí un espacio de privilegio, exclusivo en términos de producción de conocimiento, para la antropología. Proponemos, por el contrario, una voz activa para la antropología en este debate, porque ofrece herramientas para una producción más inclusiva de conocimiento.

Como señalábamos en la introducción, la idea central de este artículo ha sido plantear una reflexión sobre el tipo de conocimiento que la antropología produce y puede producir sobre el cambio climático.

24 <https://booksandideas.net/Who-owns-nature.html>.

25 Ver, por ejemplo, el argumento crítico construído a lo largo de los años por la climatóloga Judith Curry (2019). A este propósito, muy acertadamente afirma: «[la] investigación en ciencias sociales se muestra necesaria para analizar formas de incorporar la comprensión científica con todas sus incertezas en la toma de decisiones relacionadas con problemas complejos y de difícil resolución [entiéndase el cambio climático]» (Curry 2019: 39). 
Al igual que las ciencias sociales en general, la antropología al incorporar el estudio del cambio climático a su ámbito -al proponer, por ejemplo, refundar dicotomías fundacionales en las que aún se asientan muchas ciencias (especialmente la de cultura/naturaleza, legitimando divisiones disciplinares y reivindicaciones en términos de dominios) - tiene, de hecho, que establecer diálogos con otros científicos/as y desde luego etnografiarles(nos) (Bailão 2014; Parente y Curi 2017). En la antropología brasileña, por ejemplo, ha sido uno de los campos principales de abordaje de la cuestión climática: la justificación de la necesidad de las ciencias sociales (Almeida 2019) y más en particular de la antropología, en el diálogo con otras ciencias. Renzo Taddei, probablemente el autor brasileño que más ha escrito sobre clima y alteraciones climáticas $(2014,2016,2017)$, intenta definir un campo para la antropología en el ámbito del cambio climático, así como un espacio para otros especialistas (los Profetas da Chuva). Un campo que pasa por la valorización de la etnografía y de las formas de investigar que se aproxima a quienes ya están sufriendo los impactos del cambio climático o que, ya desde hace tiempo, vienen alertando contra un devenir colectivo hacia estilos y modos de vida occidentales tecnocapitalistas. Una reivindicación que vamos a encontrar también, aunque de forma más incipiente, en la antropología portuguesa (cfr. CRIA - Grupo de Investigación DASE ${ }^{26}$ ) y que en España solo ha desarrollado la sociología ${ }^{27}$.

La antropología permite acceder a la microescala de un fenómeno que, siendo global (en los impactos y en las apropiaciones políticas, filosóficas, económicas), es sobre todo en sus manifestaciones, efectos, procesos, producciones y acciones concretas, localizadas y materializadas donde debe ser conocido. $Y$ en este sentido, podemos decir que hay cuatro contribuciones principales, aunque aún embrionarias y a veces indirectas, muy importantes en los estudios antropológicos del cambio climático. Primero, al ám-

$26<$ http://cria.org.pt/wp/desafios-ambientais-sustentabilidade-e-etnografia/> (accedido el 31/3/2020). Y el texto-manifiesto de Thomas Eriksen (2016) en la revista Etnográfica, aunque en este caso, el autor lo plantea para la antropología mundial.

27 Ver la web del grupo Sociología del Cambio Climático y Desarrollo Sostenible, coordinado por Mercedes Pardo, Universidad Carlos III: <https://www.uc3m.es/ss/Satellite/ UC3MInstitucional/es/Detalle/Organismo_C/ 1371209303496/1371211779417/Sociologia_del_Cambio_ Climatico_y_Desarrollo_Sostenible>. bito de los estudios sociales de la ciencia y la tecnología, a la que ya hicimos alusión. En segundo lugar, a la crítica de las políticas públicas y de las opciones desarrollistas y extractivistas llevadas a cabo por Estados y multinacionales. Tercero, sobre los impactos y la experiencia directa del cambio climático resultante de situaciones de vulnerabilidad y de riesgo ante los desastres - un ámbito que también podríamos designar como antropología/sociología de los desastres (Taddei 2016; Santy y Valencio 2018). Aquí podemos inscribir también algunos avances conceptuales que nos posicionan en nuevas interdisciplinariedades y diálogos epistémicos (antropología - psicología - psiquiatría), como es el caso de los estudios sobre transformaciones identitarias (Dorsch 2017; Freitas 2020). Y, por fin, en el plano de las expresiones localizadas y autóctonas (conocimiento tradicional o indígena) y sus manifestaciones en términos de prácticas, percepciones, representaciones y discursos, con la vista puesta en la mitigación y la adaptación, así como en la contestación, negociación y las alternativas planteadas desde abajo, en el día a día de comunidades afectadas ( $y$ en ocasiones organizadas) por el(los) cambio(s) climático(s).

\section{BIBLIOGRAFÍA CITADA}

Acosta Naranjo, Rufino. 2002. "Ecología, santoral y rituales festivos en Pallares y su entorno». Revista de Estudios Extremeños 58(1): 259-286.

Adger, Neil. 2006. «Vulnerability». Global Environmental Change 16(3): 268-281.

Agrawal, Arun. 1995. «Dismantling the Divide between Indigenous and Scientific Knowledge». Development and Change 26(3): 413-439. doi: <https://doi.org/10.1111/j.14677660.1995.tb00560.x>.

Alexiades, Miguel. 2018. «La antropología ambiental: una visión desde el Antropoceno», en Beatriz Santamarina y Oriol Beltrán (eds.), Antropología Ambiental: Conocimientos y Prácticas Locales a las Puertas del Antropoceno: 7-70. Barcelona: Icaria.

Alexiades, Miguel y Daniela Peluso. 2016. «La urbanización indígena en la Amazonia. Un nuevo contexto de articulación social y territorial». Gazeta de Antropología 32: 1-22.

Allué, Ethel, Jordi Nadal, Alicia Estrada y Pilar García-Argüelles. 2007. "Los datos antracológicos de la Balma del Gai (Bages, Barcelona): una aportación al conocimiento de la vegetación y la explotación de los recursos forestales durante el Tardiglaciar en el NE peninsular». Trabajos de Prehistoria 64(1): 87-97. doi: <https://doi.org/10.3989/tp.2007.v64.i1.95>. 
Almeida, Jalcione. 2019. «A Sociologia e as Mudanças Climáticas». Sociologias 21(51): 9-17. doi: <https://doi.org/ 10.1590/15174522-0215100>.

Amorim, Vanessa. 2015. Marés de incerteza etnografia: do presente liminar na comunidade piscatória de Setúbal. Trabajo fin de Máster em Antropología. Lisboa: ISCTE. Disponible en: <http://hdl.handle.net/10071/10402>. Fecha de acceso: 5 may. 2020.

Araújo, Luísa y Maria João Coelho. 2013. «Políticas públicas de energia e ambiente: rumo a um país sustentável?». Sociologia, Problemas e Práticas 72: 145-158. doi: <https://doi. org/10.7458/spp2013722622>.

Arbarotti, Alexsandro y Rodrigo Martins. 2019. «Mudanças climáticas nos assentados rurais: uma etnografia sobre a experiência de futuro». Revista Retratos de Assentamentos 22(1): 116-138. doi: <https://doi.org/10.25059/2527-2594/ retratosdeassentamentos/2019.v22i1.349>.

Arora, Saurabh. 2019. «Admitting Uncertainty, Transforming Engagement: Towards Caring Practices for Sustainability beyond Climate Change». Regional Environmental Change 19(6): 1571-1584. doi: <https://doi.org/10.1007/s10113019-01528-1>.

Arora-Jonsons, Seema. 2011. "Virtue and Vulnerability: Discourses on Women, Gender and Climate Change». Global Environmental change 21(2): 744-751. doi: <https://doi. org/10.1016/j.gloenvcha.2011.01.005>

Baer, Hans A. y Merrill Singer. 2018. The Anthropology of Climate Change: An integrated critical perspective. Abingdon, Oxon; Nueva York, NY: Routledge.

Bailão, André. 2014. Ciências e Mundos aquecidos: narrativas mistas de mudanças climáticas em São Paulo. Trabajo Fin de Máster en Antropología Social. São Paulo: Universidade de São Paulo.

Barnes, Jessica, Michael Dove, Myanna Lahsen, Andrew Mathews, Pamela McElwee, Roderick McIntosh, Frances Moore et al. 2013. "Contribution of Anthropology to the Study of Climate Change». Nature Climate Change 3(6): 541-544.

Bauer, Andrew M. y Erle C. Ellis. 2018. "The Anthropocene Divide: Obscuring Understanding of Social-Environmental Change». Current Anthropology 59(2): 209-227. doi: <https://doi.org/10.1086/697198>.

Begueria, Arantza y Eva Zafra-Aparici. 2019. «Corporalidades permeables: Intersecciones entre medio ambiente y salud». AIBR. Revista de Antropología Iberoamericana 10(1): 27-48.

Biersack, Aletta. 1999. «Introduction: From the "New Ecology" to the New Ecologies». American Anthropologist 101(1): 5-18. doi: <https://doi.org/10.1525/aa.1999.101.1.5>.

Boeno, Raúl y João Ferrão. 2016. «Alterações climáticas e ordenamento do território em estratégias de segurança». Análise Social 221, LI (4.): 802-821.

Bond, Patrick y Michael K. Dorsey. 2010. «Anatomies of Environmental Knowledge \& Resistance: Diverse Climate Justice Movements and Waning Eco-Neoliberalism». Journal of Australian Political Economy (66): 286-316.
Bonfil Batalla, Guillermo. 1964. «Los que trabajan con el tiempo. Notas etnográficas sobre los graniceros de la Sierra Nevada de México». Anales de Antropología 5(1): 99-128.

Brandão, Luciana. 2019. Vidas ribeirinhas e mudanças climáticas na Amazônia: ativando híbridos, friccionando conhecimentos e tecendo redes no contexto do Antropoceno. Trabajo Fin de Máster en Sociología. Porto Alegre: Universidade Federal do Rio Grande do Sul.

Burman, Anders. 2017. "The Political Ontology of Climate Change: Moral Meteorology, Climate Justice, and the Coloniality of Reality in the Bolivian Andes». Journal of Political Ecology 24(1): 921. doi: <https://doi.org/10.2458/ v24i1.20974>.

Carabajal, María Inés. 2016. «Servicios climáticos y producción de conocimiento científico útil. Estudio de caso en una comunidad climática de Argentina». Cuardenos de Antropología Social 43: 33-49. doi: <https://doi.org/10.34096/cas. i43.1921>

Carbonell, Eliseu. 2001. "Tiempo ambiental, tiempo social. Los debates de la antropología del tiempo situados en las sociedades agrícolas del Mediterraneo, a través de la obra literaria de Josep Pla». Gazeta de Antropología 17: 24.

Carvalho, Ana. 2009. Climas de Mudança: Vulnerabilidade Humana às Alterações Climáticas no Concelho de Sintra. Trabajo Fin de Máster em Ecología Humana y Problemas Sociales Contemporaneos. Lisboa: FCSH-UNova.

Chakrabarty, Dipesh. 2009. «The Climate of History: Four Theses». Critical Inquiry 35(2): 197-222. doi: <https://doi. org/10.1086/596640>.

Cometti, Geremia. 2020. «El Antropoceno puesto a prueba en el campo: cambio climático y crisis de las relaciones de reciprocidad entre los q'ero de los Andes peruanos». Antípoda. Revista de Antropología y Arqueología 38: 3-23. doi: <https://doi.org/10.7440/antipoda38.2020.01>.

Corbacho, María Ángeles. 2011. «Sentir el viento. Las percepciones sensoriales y los vínculos con el lugar a propósito del turismo deportivo en Tarifa (Cádiz)», en Luis Díaz de Viana, Óscar Fernández Álvarez y Pedro Tomé Martín (coords.), Lugares, Tiempos, Memorias. La Antropología Ibérica en el Siglo XXI. Actas del XII Congreso de Antropología. León: FAAEE.

Correa, Sandra. 2012. «Procesos culturales y adaptación al cambio climático: la experiencia en dos islas del Caribe colombiano». Boletín de Antropología 27(44): 204-222.

Cortés Vázquez, José A. 2012. Naturalezas en conflicto. Conservación ambiental y enfrentamiento social en el Parque Natural Cabo de Gata-Níjar. Valencia: Germania \& AVA

Crate, Susan A. 2011. "Climate and Culture: Anthropology in the Era of Contemporary Climate Change». Annual Review of Anthropology 40(1): 175-194. doi: <https://doi.org/ 10.1146/annurev.anthro.012809.104925>.

Crate, Susan y Mark Nutall (eds.). 2016. Anthropology and Climate Change: From Actions to Transformations. Nueva York: Routledge. 
Crutzen, Paul J. y Will Steffen. 2003. «How Long Have We Been in the Anthropocene Era?». Climatic Change 61(3): 251-257. doi: $\quad<$ https://doi.org/10.1023/b:clim.0000004708. $74871.62>$.

Curry, Judith A. 2019. Alterações Climáticas. O que sabemos, o que não sabemos. Lisboa: Guerra e Paz.

Danowski, Deborah y Eduardo Viveiros de Castro. 2014. Há mundo por vir? ensaio sobre os medos e os fins. Florianopolis: Editora Desterro e Editora Cultura e Barbarie.

De la Villa, Ismael. 2019. «Una Nueva Ola Ecologista. ¿Puede La Lucha Contra La Crisis Climática Construir Nuevas Mayorías Sociales?». Ecología Política 58: 58-62.

Delicado, Ana, Jussara Rowland, Sofia Ribeiro, Ana Almeida, Luísa Schmidt y Susana Fonseca. 2018. Crianças e Jovens na Redução de Risco de Catástrofes. Policy Brief Observa. Lisboa: Instituto de Ciências Sociais da Universidade de Lisboa. Disponible en: <http://hdl.handle.net/10451/33337>. Fecha de acceso: 5 may. 2020.

Delicado, Ana, Luísa Schmidt, Susana Guerreiro y Carla Gomes. 2012. «Pescadores, conhecimento local e mudanças costeiras no litoral Português». Revista da Gestão Costeira Integrada 12(4): 437-451. doi: <https://doi.org/10.5894/ rgci349>

Descola, Philippe. 2013. The Ecology of Others. Chicago: Prickly Paradigm Press

Descola, Philippe y Gísli Pálsson. 2001. Naturaleza y Sociedad, Perspectivas Antropológicas. Mexico: Siglo XXI Editores.

Dias, Carlos y Stelio Marras. 2019. «Entrevista - Fala Kopenawa! Sem Floresta não tem História». Mana 25(1): 236252.

Diamond, Jared. 2006. Colapso. Por qué unas sociedades perduran y otras desaparecen. Barcelona: Random House Mondadon.

Diz, Carlos. 2017. "La ciudad narrada: Revuelta y semántica del conflicto urbano». Inmediaciones de la Comunicación 12(1): 191-213. doi: <https://doi.org/10.18861/ic.2017. $12.2673>$.

Djoudi, Houria, Bruno Locatelli, Chloe Vaast, Kiran Asher, Maria Brockhaus y Bimbika Basnett Sijapati. 2016. «Beyond dichotomies: Gender and intersecting inequalities in climate change studies». Ambio 45(3): 248-262. doi: <https://doi. org/10.1007/s13280-016-0825-2>.

Dorsch, Laura. 2017. A objectificação da cultura Sámi: adaptabilidade no tempo e reconhecimento da identidade no norte da Sápmi norueguesa. Trabajo Fin de Máster. Lisboa: ISCTE-IUL. Disponible en: <http://hdl.handle.net/ 10071/14594>. Fecha de acceso: 5 may. 2020.

Dove, Michael (ed.). 2014. The Anthropology of Climate Change. An Historical Reader. Malden, Oxford y West Sussex: Wiley Blackwell.

Dunlap, Alexander y Sian Sullivan. 2019. «A Faultline in Neoliberal Environmental Governance Scholarship? Or, Why Accumulation-by-Alienation Matters». Environment and
Planning E: Nature and Space 3(2): 552-579. doi: <https:// doi.org/10.1177/2514848619874691>.

Emmett, Robert y Thomas Lekan. 2016. Whose Anthropocene? Revisiting Dipesh Chakrabarty's "Four Theses". Vol. 2. RCC Perspectives: Transformations in Environment and Society.

Eriksen, Thomas Hylland. 2016. «Sobreaquecimento: pequenos lugares e grandes questões na antropologia do século XXI». Etnográfica 20(1): 197-208. doi: <https://doi. org/10.4000/etnografica.4204>.

Escobar, Arturo. 2007. La invención del Tercer Mundo: Construcción y deconstrucción del desarrollo. Caracas: Fundación Editorial el perro y la rana.

Escobar, Arturo. 2016. «Sentipensar con la Tierra: Las Luchas Territoriales y la Dimensión Ontológica de las Epistemologías del Sur». AIBR. Revista de Antropología Iberoamericana 11(1): 11-32

Faulhaber, Priscila. 2004. "As estrelas eram terrenas": antropologia do clima, da iconografia e das constelações Ticuna». Revista de Antropologia 47(2): 379-426. doi: <https:// doi.org/10.1590/s0034-77012004000200002>.

Felli, Romain. 2019. «Beyond the Critique of Carbon Markets: The Real Utopia of a Democratic Climate Protection Agency». Geoforum 98 (January): 236-243. doi: <https://doi. org/10.1016/j.geoforum.2018.02.031>.

Fernández Llamazares, Álvaro, Raquel Amaral García, Isabel Díaz-Reviriego, Mar Cabeza, Aili Pyhälä y Victoria ReyesGarcía. 2017. «An Empirically Tested Overlap between Indigenous and Scientific Knowledge of a Changing Climate in Bolivian Amazonia», Regional Environmental Change 17(6): 1673-1685. doi: <https://doi.org/10.1007/s10113017-1125-5>.

Ferrão, João y Ana Delicado (coord.). 2019. Portugal Social em Mudança: Objectivos do Desenvolvimento Sustentável. Lisboa: Instituto de Ciências Sociais da Universidade de Lisboa.

Fiske, Shirley, Susan Crate, Carole Crumley, Kathleen Galvin, Heather Lazrus, George Luber, Lisa Lucero et al. 2014. Changing the Atmosphere. Anthropology and Climate Change. Final Report of the AAA Global Climate Change Task Force. Arlington, VA: American Anthropological Association.

Fletcher, Robert. 2012. «Capitalizing on Chaos: Climate Change and Disaster Capitalism». Ephemera 12(1/2): 97-112.

Fleury, Lorena, Jean Miguel y Renzo Taddei. 2019. «Mudanças climáticas, ciência e sociedade». Sociologias 51: 18-42. doi: <https://doi.org/10.1590/15174522-0215101>.

Forsyth, Tim. 2014. «Climate Justice Is Not Just Ice». Geoforum 54 (July): 230-232. doi: <https://doi.org/10.1016/j. geoforum.2012.12.008>.

Foster, John Bellamy, Brett Clark y Richard York. 2009. «The Midas Effect: A Critique of Climate Change Economics: A Critique of Climate Change Economics». Development and Change 40(6): 1085-1097. doi: <https://doi.org/10.1111/ j.1467-7660.2009.01613.x> 
Franquesa, Jaume. 2018. Power Struggles: Dignity, Value, and the Renewable Energy Frontier in Spain. Bloomington: Indiana University Press.

Fraser, Nancy. 2000. «Rethinking Recognition». New Left Review 3(May.-Jun.): 107-120.

Freitas, Márcio. 2020. A terceira margem do rio: conflitos ontológicos no litoral norte capixaba, Tesis doctoral, Lisboa: NOVA-FCSH.

Frömming, Urte Undine. 2009. «Kilimanjaro's Melting Glaciers: on the Colonial and Postcolonial Perception and Appropriation of African Nature». Etnográfica 13(2): 395-416.

Freeman, Derek, 1983. Margaret Mead and Samoa: The Making and Unmaking of an Anthropological Myth. Cambridge: Harvard University Press.

Galván Tudela, José Alberto y Aimet Guevara Labaut. 2014. «Saberes etnoecológicos y gestión local del impacto del huracán Sandy en Contramaestre (Santiago de Cuba): una mirada desde las religiones afrocubanas», en Joan Prats (coord.), Periferias, Fronteras y Diálogos. Actas del XIII Congreso de Antropología. Tarragona: Universitat Rovira i Virgili

García del Amo, David, Graham Mortyn y Victoria Reyes-García. 2020. «Including Indigenous and Local Knowledge in Climate Research: an Assessment of the Opinion of Spanish Climate Change Researchers». Climatic change 160: 67-88. doi: <https://doi.org/10.1007/s10584-019-02628-x>.

Gellner, Ernest. 1988. Plough, Sword and Book. The Structure of Human History. Chicago: University of Chicago Press.

Gibson, Hannah y Sita Venkateswar. 2015. «Anthropological Engagement with the Anthropocene: A Critical Review». Environment and Society 6(1): 5-27. doi: <https://doi. org/10.3167/ares.2015.060102>.

Godden, Lee y Maureen Tehan. 2016. «REDD+: Climate Justice and Indigenous and Local Community Rights in an Era of Climate Disruption». Journal of Energy and Natural Resources Law 34(1): 95-108. doi: <https://doi.org/10.1080/ 02646811.2016.1121620>.

Goloubinoff, Marina, Ester Katz y Annamaria Lammel. 1997. Antropología del clima en el mundo hispanoamericano (Tomo I y II). Quito: Abya-Yala.

Gomes, Carla. 2019. "Sustainability Challenges for Sub-Saharan Africa: Vulnerability, Justice and Human Capabilities», en Manuel Guedes y Gustavo Cantuaria (eds.), Bioclimatic Architecture in Warm Climates: 285-297. Cham, Suiza: Springer.

González Abrisketa, Olatz y Susana Carro Ripalda. 2016. «La apertura ontológica en la antropología contemporánea». Revista de Dialectología y Tradiciones Populares 71(1): 101128. doi: <https://doi.org/10.3989/rdtp.2016.01.003>.

González Concepción, Óscar y Alfredo Rubio Pérez. 2014. «Cambio climático y la percepción de los campesinos sobre la variedad café robusta en la zona de La Torcaza, Sierra Maestra, Cuba». Batey: Revista cubana de Antropología Sociocultural 6(6): 110-119.
González Martínez, Sandra Llovizna, Luis Arturo Ávila Meléndez, Gustavo Blanco Wells y José Teodoro Silva García. 2015. «Comunidades indígenas: Entre la adaptación a alteraciones climáticas locales y el abandono de la agricultura». AIBR, Revista de Antropología Iberoamericana 10(1): 27-48.

Grau-Satorras, Mar, lago Otero, Erik Gómez-Baggetun y Victoria Reyes-García. 2016. «Long-term Community Responses to Droughts in the Early Modern Period: The Case Study of Terrassa, Spain ». Ecology and Society 21(2): artículo 33. doi: <https://doi.org/10.5751/es-08232-210233>.

Guerra, João, Ana Horta, Madalena Santos y Luísa Schmidt. 2019. "Alterações Climáticas», en João Ferrão y Delicado, Ana (coord.), Portugal Social em Mudança: Objectivos do Desenvolvimento Sustentável: 37-45. Lisboa: Instituto de Ciências Sociais da Universidade de Lisboa.

Haouari, Mohamed. 2014. «Experiencias de trabajo social en el norte de Marruecos con grupos vulnerables en tiempos de crisis». Gazeta de Antropología, 2014, 30 (2), artículo 06. Disponible en: <http://hdl.handle.net/10481/33425>.

Haraway, Donna J. 1991. Simians, Cyborgs, and Women. The Reinvention of Nature. Nueva York: Routlege.

Haraway, Donna J. 2015. "Anthropocene, Capitalocene, Plantationocene, Chthulucene: Making Kin». Environmental Humanities 6(1): 159-165. doi: <https://doi.org/10.1215/ 22011919-3615934>.

Hastrup, Kirsten y Martin Skrydstrup. 2013. The Social Life of Climate Change Models: Anticipating Nature. Nueva York, Londres: Routledge.

Hémond, Aline y Marina Goloubinoff. 1997. «El camino de cruz del agua: Clima, calendario agrícola y religioso entre los nahua de Guerrero (México)», en Marina Goloubinoff, Ester Katz y Annamaria Lammel, Antropología del clima en el mundo hispanoamericano (Tomo / y II): 237-274. Quito: Abya-Yala.

Heyd, Thomas. 2011. «Pensar la relación entre cultura y cambio climático», en Astrid Ulloa (ed.), Perspectivas culturales del clima: 17-32. Bogotá: Universidad Nacional de Colombia.

Ingold, Tim. 2000. Perception of the Environment. Essays on Livelihood, Dwelling and Skill. Londres: Routledge.

IPCC. 2018. Global Warming of $1.5^{\circ} \mathrm{C}$. An IPCC Special Report on the Impacts of Global Warming of $1.5^{\circ} \mathrm{C}$ above Pre-industrial Levels and Related Global Greenhouse Gas Emission Pathways, in the Context of Strengthening the Global Response to the Threat of Climate Change, Sustainable Development, and Efforts to Eradicate Poverty [Masson-Delmotte, V., P. Zhai, H.-O. Pörtner, D. Roberts, J. Skea, P. R. Shukla, A. Pirani et al. (eds.)]. Disponible en: <https://www.ipcc.ch/ sr15/>.

Jafry, Tahseen. 2019. Routledge Handbook of Climate Justice. New York, London: Routledge.

Jasanoff, Sheila. 2004. States of Knowledge. The Co-Production of Science and Social Order. London y New York: Routledge.

Katz, Esther. 1997. «Ritos, representaciones y meteorología en la "Tierra de la Lluvia" (Mixteca, México)», en Marina Goloubinoff, Ester Katz y Annamaria Lammel, Antropología del 
clima en el mundo hispanoamericano (Tomo / y II): 99-134. Quito: Abya-Yala.

Katz, Esther y Annamária Lammel. 2008. «Elementos para una antropología del clima», en Annamária Lammel, Marina Goloubinoff y Esther Katz (eds.), Aires y Lluvias. Antropología del Clima en México: 27-50. México: CIESAS, IRD, Centro de Estudios Mexicanos y Centroamericanos.

Kellogg, William y Margaret Mead. 1980. The Atmosphere: Endangered and Endangering. Tunbridge Wells: Castle House Publications.

Keucheyan, Razmig. 2014. La nature est un champ de bataille. Essai d'écologie politique. Paris: La Découverte.

Klein, Naomi. 2014. This Changes Everything: Capitalism vs. the Climate. Toronto: Alfred A. Knopf Canada.

Kopenawa, Davi y Bruce Albert. 2013. The Falling Sky. Words of a Yanomami Shaman. Cambridge y London: Harvard University Press.

Kosanic, Alexandra, Jan Petzold, Amy Dunham y Mialy Razanajatovo. 2019. "Climate Concerns and the Disabled Community». Science (Letters) 366(6466). doi: <https://doi. org/10.1126/science.aaz9045>.

Latour, Burno. 1993. We Have Never Been Modern. London, Prentice Hall.

Litre, Gabriela y Marcel Bursztyn. 2015. «Percepções e Adaptação aos Riscos Climáticos e Socioeconómicos na Pecuária Familiar do Bioma Pampa». Ambiente \& Sociedade XVIII(3): 55-80. doi: <https://doi.org/10.1590/18094422asoc668v1832015>.

Lobo, Rodrigo. 2016. Naturezas esfumaçadas: os Tembé e o mercado de crédito de carbono. Tesis Doctoral, Doctorado en Antropología Social, São Paulo: Universidade São Paulo.

Madaleno, Isabel. 2012. «O povo que mede forças com a morte: os ilhéus de Tuvalu, no Pacífico Sul, e a subida das águas do mar». Boletim do Museu Paraense Emílio Goeldi. Ciencias Humanas 7(2): 493-508. doi: <https://doi.org/10.1590/ s1981-81222012000200011>.

Marconetto, Bernarda y Mariano Bussi. 2018. «Fines de mundos "otros". Seca y sequía en conflicto". Chungara Revista de Antropología Chilena 50(2): 319-329. doi: <https://doi. org/10.4067/s0717-73562018005000901>.

Mendes, Paulo. 2013. O Mar é Que Manda. Comunidade e Percepção do Ambiente no Litoral Alentejano. Lisboa, Fundação Calouste Gulbenkian, FCT.

Mesquita, Erika. 2012. Ver de perto pra contar de certo: as mudanças climáticas sob os olhares dos moradores da floresta do Alto do Juruá. Tesis de Doctorado. Campinas: Universidade Estadual de Campinas (UNICAMP).

Mesquita, Erika. 2015. «Mudanças climáticas e a cosmovisão Ashaninka». ClimaCom 3: 56-61.

Miguel, Jean y Marko Monteiro. 2014. «Por que devemos nos interessar por modelos climáticos?». ClimaCom 1: 6-16.

Muradian, Roldan, Esteve Corbera, Unai Pascual, Nicolás Kosoy y Peter H. May. 2010. «Reconciling Theory and Practice: An
Alternative Conceptual Framework for Understanding Payments for Environmental Services». Ecological Economics 69(6): 1202-1208. doi: <https://doi.org/10.1016/j. ecolecon.2009.11.006>

Nates-Cruz, Beatriz y Patricia Serón. 1997. «"El tiempo que hace": Percepción de los fenómenos meteorológicos entre los paeces (Colombia)», en Marina Goloubinoff, Ester Katz y Annamaria Lammel, Antropología del clima en el mundo hispanoamericano (Tomo I y II): 57-82. Quito: Abya-Yala.

Ordóñez Vergara, Pilar. 1993. «Los molineros de La Alpujarra». Gazeta de Antropología, 1993, 10, artículo 13. Disponible en: <http://hdl.handle.net/10481/13642>.

Pardo Buendía, Mercedes. 2007. «El impacto social del cambio climático». Panorama Social 5(Primer Semestre): 22-35.

Parente, Izabel y Melissa Curi. 2017. «Um estudo sobre o estado da arte da Antropologia do Clima». Bib 80: 42-58.

Pascual, Unai, Jacob Phelps, Eneko Garmendia, Katrina Brown, Esteve Corbera, Adrian Martin, Erik Gomez-Baggethun y Roldan Muradian. 2014. "Social Equity Matters in Payments for Ecosystem Services». BioScience 64(11): 1027-1036. doi: <https://doi.org/10.1093/biosci/biu146>

Paz, Josi. 2012. O clima do consumo: a sociedade de consumidores no debate sobre a mudança climática. Tesis Doctoral, Doctorado en Sociologia, Brasília: Universidade de Brasília.

Pettit, Jethro. 2004. «Climate Justice: A New Social Movement for Atmospheric Rights». IDS Bulletin 35(3): 102-106. doi: <https://doi.org/10.1111/j.1759-5436.2004.tb00142.x>.

Pokrant, Bob. 2016. «Climate Change Adaptation and Development Planning. From Resilience to Transformation?», en Helen Kopnina y Eleanor Shoreman-Ouimet, Routledge Handbook of Environmental Anthropology: 242-255. Londres y Nueva York: Routledge.

Rapapport, Roy, 1979. Ecology, Meaning and Religion. Richmond: North Atlantic Books.

Ravera, Federica e Irene Iniesta Arandia. 2017. «Perspectivas feministas para repensar la investigación en cambio climático y las políticas de adaptación». Ecología Política 53: 4144.

Ravera, Federica, Victoria Reyes-García, Unai Pascual, Adam Drucker, David Torrasón y Mauricio Bellón. 2019. «Gendered Agrobiodiversity Management and Adaptation to Climate Change: Differentiated Strategies in Two Marginal Rural Areas of India». Agriculture and Human Values 36(3): 455474. doi: <https://doi.org/10.1007/s10460-018-09907-w>.

Rayner, Steve y Elisabeth Malone. 1998. Human Choice and Climate Change: An International Assessment. Columbus: Ballete Press.

Reyes García, Victoria, David García del Amo, Petra Benyei, Álvaro Fernández Llamazares, Konstatina Gravani, André Junqueira, Vanesse Labeyrie et al. 2019. «A Collaborative Approach to Bring Insights from Local Observations of Climate Change Impacts into Global Climate Change Research». Current Opinion in Environmental Sustainability 39: 1-8. doi: <https://doi.org/10.1016/j.cosust.2019.04.007>. 
Ribeiro, Walmeri. 2015. "Territórios Sensíveis: uma investigação performativa em arte, ciência, tecnologia e natureza». ARS 13(26): 204-213.

Rodríguez Mir, Javier. 2011. «Transformando la alteridad. Alcances y limitaciones de los proyectos de desarrollo en las sociedades originarias del Chaco argentino». Gazeta de Antropología, 2011, 27 (1), artículo 17. Disponible en: <http://hdl.handle.net/10481/17497>.

Roque de Pinho, Joana. 2013. "Shooting Climate Change in the Maasai Mara: Aesthetics and Expectations in Participatory Filmmaking with Kenyan Pastoralists». Anthropology Now 5(2): 74-86. doi: <https://doi.org/10.1080/19492901.2013. 11728404>.

Roque de Pinho, Joana y Kathleen Galvin. 2015. «Maasai Voices on Climate Change (and other changes too): Participatory video and communication about environmental changes in the East African rangelands", en Maria Brites, Ana Jorge, Sílvio Santos (eds.), Metodologias participativas: Os media e a educação: 235-248. Covilhã: LabCom Books.

Rosario Molina, Juan Carlos. 2014. «Percepciones del tiempo climático en las poblaciones campesinas de Contramaestre, Santiago de Cuba». Batey: Revista Cubana de Antropología Sociocultural 6(6): 98-109.

Rosewarne, Stuart, James Goodman y Rebecca Pearse. 2014. Climate Action Upsurge: The Ethnography of Climate Movement Politics. Nueva York: Routledge.

Rossbach de Olmos, Lioba. 2014. «El cambio climático y la antropología: perspectivas culturales sobre un problema ambiental global», en Joan Prats (coord.) Periferias, Fronteras y Diálogos. Actas del XIII Congreso de Antropología: 2982-2999. Tarragona: Universitat Rovira i Virgili.

Rossbach de Olmos, Lioba. 2019. «Manejando incompatibilidades. El sistema de adivinación de ifá y el cambio climático en Cuba». Anthropos 114: 33-36.

Rossbach de Olmos, Lioba y Ernst Halbmayer. 2014. "Clima, atmósfera y ambiente: una pregunta más allá de las ciencias naturales». Batey: Revista Cubana de Antropología Sociocultural 6(6): 3-21.

Santamarina Campos, Beatriz. 2008. «Antropología y Medio Ambiente. Revisión de una tradición y nuevas perspectivas de análisis en la problemática ecológica». AIBR. Revista de Antropología Iberoamericana 3(2): 144-184.

Santamarina, Beatriz. 2012. "La construcción del cambio climático en la comunidad valenciana. (Algunas notas sobre imprecisiones, contradicciones y desplazamientos)", en Artemio Baigorri (ed.), Transiciones Ambientales. Ensayos de Sociología Ambiental: 79-104. ARS Sociológica.

Santos, Madalena. 2017. Análise da decisão portuguesa de atribuição de concessões para a pesquisa e prospecção de petróleo na costa algarvia à luz do quadro europeu de combate às alterações climáticas. Trabajo de Fin de Master en Antropología. Lisboa: ISCTE-IUL.

Santos, Boaventura de Sousa y Maria Paula Meneses (orgs.). 2010. Epistemologias do Sul. São Paulo: Editora Cortez.
Santy, Boaventura y Norma Valencio. 2018. «Políticas de Adaptação às Mudanças Climáticas e a Produção Transescalar da Sujeição Social na Guiné-Bissau». Cadernos de Estudos Africanos 36: 161-185. doi:<https://doi.org/10.4000/cea.3308>.

Sapiains, Rodolfo, Ana M. Ugarte y Julio Hasbún. 2019. «Percepciones del cambio climático en la isla de chiloé: desafíos para la gobernanza local». Magallania 47(1): 83-103. doi: <https://doi.org/10.4067/s0718-22442019000100083>.

Schlosberg, David y Lisette B. Collins. 2014. «From Environmental to Climate Justice: Climate Change and the Discourse of Environmental Justice: Climate Change and the Discourse of Environmental Justice». Climate Change 5(3): 359-374. doi: <https://doi.org/10.1002/wcc.275>.

Schmidt, Luísa, Adriana Alves, João Guerra y João Mourato. 2018. «Perceções sociais e estratégias locais de adaptação às Alterações Climáticas: a experiência do projeto ClimAdaPT.Local», en Miranda, A. I., M. Lopes, L. Tarelho, F. Martins, P. Roebeling, M. Coelho, J. Labrincha (eds.), Actas da Conferência Internacional de Ambiente em Língua Portugesa (CIALP 2018): I, 287-296. Aveiro: Departamento de Ambiente e Ordenamento Universidade de Aveiro.

Schmidt, Luísa, Filipe Duarte Santos, Pedro Prista, Tiago Saraiva y Carla Gomes. 2012, "Alterações Climáticas, Sociais e Políticas em Portugal: Processos de Governança num litoral em Risco». Ambiente \& Sociedade XV(1): 23-40. doi: <https://doi.org/10.1590/s1414-753×2012000100003>.

Seixas, Sônia, João Hoeffel, Michelle Renk, Benedita Silva y Fábio Lima. 2014. "Percepção de pescadores e maricultores sobre mudanças ambientais globais, no litoral Norte Paulista, São Paulo, Brasil». Revista da Gestão Costeira Integrada 14(1): 51-64. doi: <https://doi.org/10.5894/rgci424>

Shankman, Paul. 2009. The Trashing of Margaret Mead. Anatomy of an Anthropological Controversy. Madison, University of Wisconsin Press.

Sillitoe, Paul. 1993. "A Ritual Response to Climatic Perturbations in the Highlands of Papua New Guinea». Ethnology 32(2): 169-185. doi: <https://doi.org/10.2307/3773771>.

Singer, Merrill. 2019. Climate Change and Social Inequality : The Health and Social Costs of Global Warming. Abingdon, Oxon; Nueva York: Routledge.

Soares, Denise y Antonino García. 2014. «Percepciones campesinas indígenas acerca del cambio climático en la cuenca de Jovel, Chiapas - México». Cuadernos de Antropología Social 39: 63-89.

Soares Moraes, Denise y Sergio Vargas Velázquez. 2012. "Capitales comunitarios y vulnerabilidad social frente al cambio climático en un municipio de Yucatán». Trayectorias 14(3334): 51-75.

Steward, Julian H. 1938. "Ecological aspects of southwestern society». Anthropos 32: 87-104. Disponible en: <https:// www.jstor.org/stable/40447907>.

Swyngedouw, Erik. 2010. «Apocalypse Forever?». Theory, Culture \& Society 27(2-3): 213-232. doi: <https://doi.org/ $10.1177 / 0263276409358728>$. 
Taddei, Renzo. 2014. «As secas como modos de enredamento». ClimaCom 1: 36-41.

Taddei, Renzo. 2016. "Os desastres em uma perspectiva antropológica». ComCiência, dossiê 219. Disponible en: <http://www.comciencia.br/comciencia/?section=8\& edicao=121\&id=1469\&print=true $>$. Fecha de acceso: 5 may. 2020 .

Taddei, Renzo. 2017. Meteorologistas e Profetas da Chuva. Conhecimentos, práticas e políticas da atmosfera. Bela Vista-São Paulo: Terceiro Nome.

Taddei, Renzo y Sophie Haines. 2019. «Quando climatologistas encontram cientistas sociais: especulações etnográficas sobre equívocos interdisciplinares». Sociologias 21(51): 186209.

Temper, Leah. 2019. «Blocking Pipelines, Unsettling Environmental Justice: From Rights of Nature to Responsibility to Territory». Local Environment 24(2): 94-112. doi: <https:// doi.org/10.1080/13549839.2018.1536698>

Tocancipá-Falla, Jairo, Javier Ernán Rosero y Carlos Felipe Restrepo. 2011. «Percepciones, representaciones religiosas y conocimiento local sobre el clima y sus cambios en el $\mathrm{Pa}$ cífico caucano, Colombia», en Astrid Ulloa (ed.), Perspectivas culturales del clima: 395-426. Bogotá: Universidad Nacional de Colombia.

Tokar, Brian. 2019. «On the Evolution and Continuing Development of the Climate Justice Movement», en Tahseen Jafry (ed.), Routledge Handbook in Climate Justice: 13-25. Abingdon, Oxon; Nueva York, NY: Routledge.

Ulloa, Astrid. 2010. "Geopolíticas del cambio climático». Anthropos 227: 133-146.

Ulloa, Astrid (ed.). 2011. Perspectivas culturales del clima. Bogotá: Universidad Nacional de Colombia.

Ulloa, Astrid. 2014. «Dimensiones culturales del clima: Indicadores y predicciones entre pobladores locales en Colombia». Batey: Revista Cubana de Antropología Sociocultural 6:17-33

Ulloa, Astrid. 2015. "Produção de conhecimentos sobre o clima. Processos históricos de exclusão/ apropriação de saberes e territórios de mulheres e povos indígenas». ClimaCom 3: 34-52.

Ulloa, Astrid. 2016. "Justicia climática y mujeres indígenas en América Latina». Latin American Studies Association ForumLASAFORUM 47(4): 12-16.
Ulloa, Astrid. 2018. «Reconfiguring Climate Change Adaptation Policy: Indigenous Peoples' Strategies and Policies for Managing Environmental Transformations in Colombia», en Silja Klepp y Libertad Chávez-Rodríguez (eds.), A Critical Approach to Climate Change Adaptation: Discourses, Policies and Practices: 222-237. Abindgon, Oxon; Nueva York: Routledge.

Urry, John. 2011. Climate Change and Society. Cambridge: Polity.

Vázquez Palacios, Felipe y Rodrigo Tovar Cabañas. 2018. "Las incertidumbres sobre el cambio climático y el apocalipsis en los creyentes de iglesias Judeo-cristianas en México». Batey: Revista Cubana de Antropología Sociocultural 11(2): 81-95.

Ventura, Artur. 2008. Antropologia do ambiente: um contributo para o estudo das alterações climáticas: entre os discursos, as percepções dos riscos, e as práticas quotidianas numa amostra da população da freguesia de Alcântara. Dissertação de Mestrado. Lisboa: Instituto Superior de Ciências Sociais e Políticas.

Vicent García, Juan Manuel, M.a Isabel Martínez Navarrete, José Antonio López Sáez e Ignacio de Zavala Morencos. 2010. «Impacto medioambiental de la minería y la metalurgia del cobre durante la Edad del Bronce en Kargaly (región de Orenburgo, Rusia)». Trabajos de Prehistoria 67(2): 511-544. doi: <https://doi.org/10.3989/tp.2010.10054>.

Villalobos Acosta, César. 2018. «Cambio climático y arqueología en el desierto de Sonora». Anales de Antropología 52(2): 37-53. doi: <https://doi.org/10.22201/iia.24486221e.2018. 2.62470>.

Villela, Samuel. 1997. «Vientos, nubes, Iluvias, arcoiris: Simbolización de los elementos naturales en el ritual agrícola de la Montaña de Guerrero (México)», en Marina Goloubinoff, Ester Katz y Annamaria Lammel (eds.), Antropología del clima en el mundo hispanoamericano (Tomo I y II): 225-236. Quito: Abya-Yala.

Viveiros de Castro, Eduardo. 1998. "Cosmological Deixis and Amerindian Perspectivism». The Journal of the Royal Anthropological Institute 4(3): 469. doi: <https://doi. org/10.2307/3034157>.

Welch-Devine, Meredith, Anne Sourdril y Brian J. Burke (eds.). 2020. Changing Climate, Changing Worlds: Local Knowledge and the Challenges of Social and Ecological Change. Suiza: Springer. 